$1-1-1893$

\title{
Catalogue of West Virginia forest and shade tree insects
}

\author{
A. D. Hopkins
}

Follow this and additional works at: https://researchrepository.wvu.edu/ wv_agricultural_and_forestry_experiment_station_bulletins

\section{Digital Commons Citation}

Hopkins, A. D., "Catalogue of West Virginia forest and shade tree insects" (1893). West Virginia Agricultural and Forestry Experiment Station Bulletins. 32.

https://researchrepository.wvu.edu/wv_agricultural_and_forestry_experiment_station_bulletins/32 @ WVU. It has been accepted for inclusion in West Virginia Agricultural and Forestry Experiment Station Bulletins by an authorized administrator of The Research Repository @WVU. For more information, please contact ian.harmon@mail.wvu.edu. 



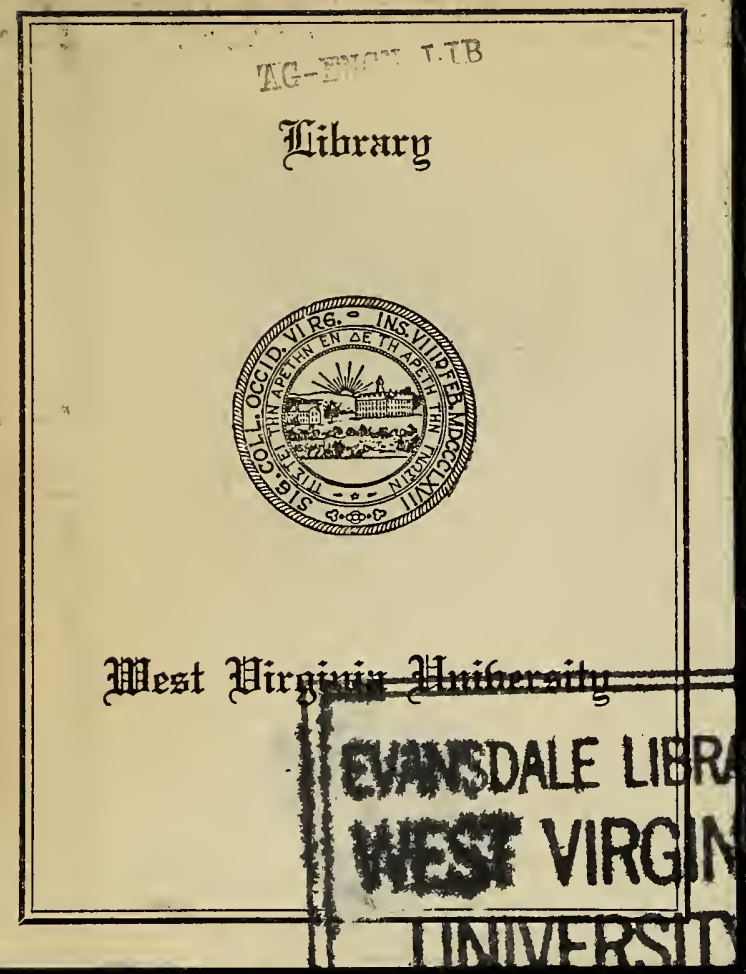


West Virginia University Libraries
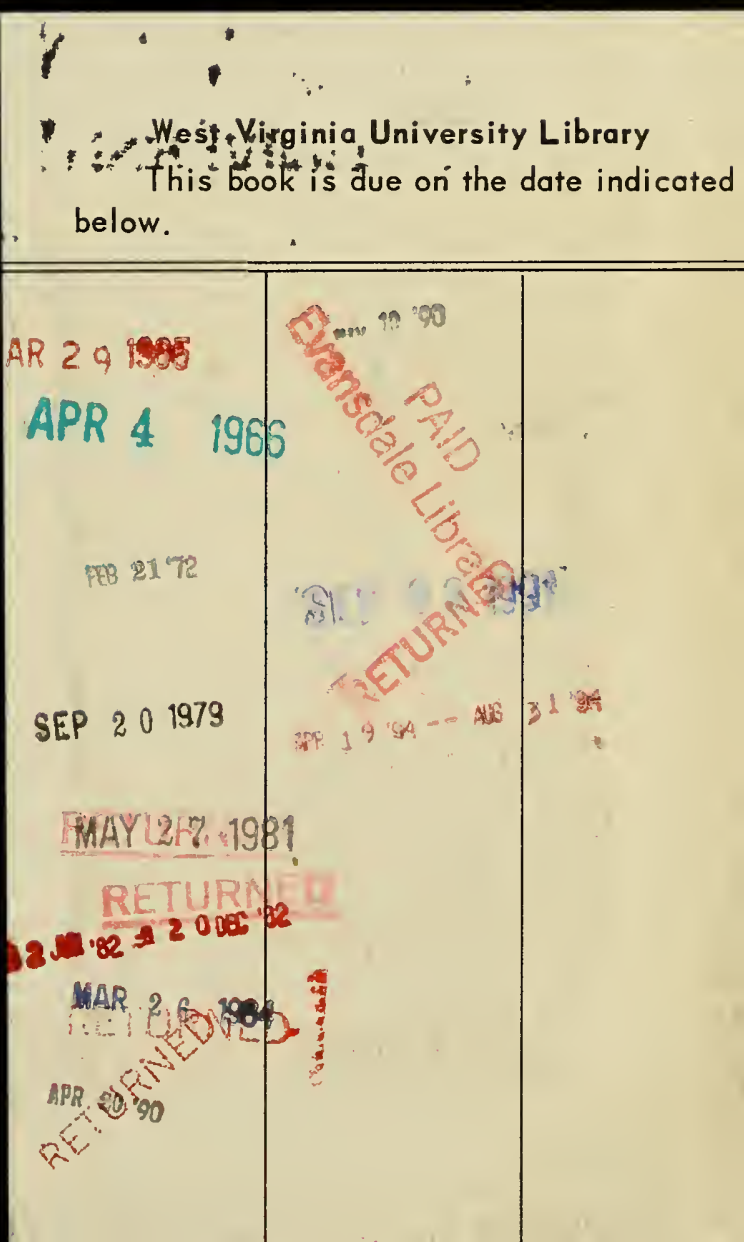
Digitized by the Internet Archive in 2010 with funding from

Lyrasis Members and Sloan Foundation 


\title{
Bulletin No 32 \\ WFSI VIRGINIA
}

Agricultural Experiment Station

\author{
MURGANTOWN, W VA.
}

CATALOGUE

OF

West Virginia Forest and Shade Tree INSECTS.

$$
\text { MA Y }, 1893 .
$$

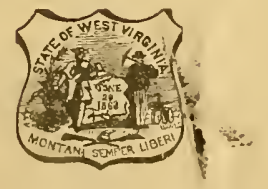

E'HARLESTON. W. YA. 


\title{
BOARD OF REGENTS OF THE WEST VIRGINIA UNIVERSITY.
}

\begin{tabular}{|c|c|c|}
\hline District. & Name of Regent. & P. O. Address. \\
\hline 1. & J. B. SUMMERVILLE, & Wheeling. \\
\hline 2. & CUARENCE L. SMITH, & Fairmont. \\
\hline 3. & R. G. LYNN, & Glenville. \\
\hline 4. & JOHN C. VANCE, & Clarksburg, \\
\hline 5. & JOHN G. SCHILLING, & Spencer. \\
\hline 6 . & EDWARD A. BENNETT, & Huntington, \\
\hline 7 . & WIRT A. FRENCH, & l'rinceton, \\
\hline S. & M. J KESTER, & Union. \\
\hline प. & J. F. BROWN, & Charleston. \\
\hline 10. & THOS. J FARNSWORTH, & BuckLannon. \\
\hline 11. & JOSEPH MORELAND, & Morgantown. \\
\hline 12. & JOHN A. ROBINSON, & Patterson's Depot. \\
\hline & & Kahletown. \\
\hline
\end{tabular}

\section{MEMBERS OF THE STATION COMMITTEE.}

\author{
JOHN A ROBINSON, \\ JOSEPH MORELAND, \\ C I SMITH, \\ J. B. SOMMER VILLE,
} DR. W. W. BROWN.

Prest. of Boakd of Regen's,

President of tile University,

E. M. TURNER, LL. D.,
JOHN $\Lambda$ ROBINSON.

Treasurer,

JOHN I. HARVEY.

\section{STATION STAFF.}

JOHN A. MYERS, Pri D, F. Wr. RANE, M S.

A. D. HOPKTNS,

D. D. JOHNSON, A. M.,. RUDOLF DE ROODE, l'Ir. D), SUSIE V. MAYERS,
Director. Horticulturist and Microscopist. Rntomologist. Agriculturist. Chemist. Stenographer and Book-keeper. 


\title{
C A T A L O G U E.
}

\section{OF}

\section{WEST VIRGINIA FOREST AND SHADE TREE IN- SECTS.}

Collected in I890-1893, Including Injurions, Beneficial and other Insects Taken on or in Some Part of the Tree Examined.

\author{
BY A. I). HOPKINS.
}

INTRODUCRORY*.

'The depredations by insects on certain forest and shade trees of West Virginia during the past three years have been such as to demand special investigation, with a view of finding the species responsible for the primary cause of the premature death of trees; also to discover, if possible, methods of repelling or destroying them.

Insects injurious and beneficial to the Yellow Locust, the Black Spruce and the Pines have claimed the greater share of my attention, yet at the same time, such insects as were found on or in other kinds of trees and shrubs were collected and their habits, so far as observed, noted.

These investigations have not, as yet, been sufficiently completed to justify a detailed report of the work and results; therefore this catalngue is sent out in alvance of more popular bulletins on the subject to be issued at the end of another season's work.

This catalogue and the one preceding it on IVest Virginia Scolyticle are issued in this form, and in small editions not for general distribution, but for those who may be interested in such records of original observation, on the habits and life histories of forest, shade and fruit tree insects

The species herein mentioned have been collected or bred by me during the past three yeass, and the reference to habits, dates, etc., are firom my notes and recorks.

It is my intention to continue the study of the life histories of forest tree insects, and to publish from time to timo supplements to these catalogues containing lists of adclitional species found, together with necessary corrections and revisions.

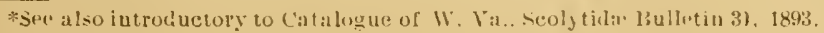


My time being devoted almost exclusively to the study of insects from an economic standpoint, I have little time for systematic study, except on the family Scolytidæ; therefore I shall, as heretofore, depend largely upon the authoritative specialists for determinations of species.

I desire, in this connection, to acknowledge the kindness of the following named persons who have made first determinations for me of species in orders and families in which they are specialists: Dr. C. V. Riley, Mr. L. O. Howard, Mr. William Ashmead, Mr. E A. Schwarz, of the Division of Entomology at Washington; Dr. G. H. Horn, of Philadelphia, Dr. W. J. Holland, of l'ittshurg; Mr. W. H. Edwards, of Coalburg, W Va.; Prof C. H. Tyler Townsend, of Los Cruces, N. M. ; and Oberforester W. Eichhoff, of Strassburg, Germany.

\section{Exhibit Collections.}

Two exhibit collections have been prepared during the past winter. One for the exhibit by the Association of Agricultural Colleges and Experiment Stations in the Agricultural Building; the other for the West Virginia Exhibit in the Forestry Building at the World's Columbian Exposition. The former consists of West Virginia Scolytidæ and some of their enemies, and contains 299 insects and 284 wood and bark specimens, showing character of damage to wood and bark of forest, shade, fruit trees, etc. The latter consists of West Virginia forest and shade tree insects and examples of their work in wood and bark, including 240 insects and 250 other specimens. The species numbered in heavy type in this catalogue are represented in this collection.

These exhibits, aside from indicating the character of work in Entomology carried on by the West Virginia Experiment Station are designed to call the attention of owners of timber and others, who are interested in the preservation of our forests, to the importance of earnest and careful investigations, with a view of becoming better acquainted with the habits and life histories of the insects which cause such great damage and loss. 


\section{Order COLEOPTERA; Family CARABID FE.}

1. Cychrus Ridingsii, Bland.

Adult September 2.

Collected under bark on spruce log. Randolph county.

2. Tachys nanus, Gyll.

Adult September 1.

Collected under bark on spruce log

Randolph county.

3 Pterostichus adoxus, Say.

Collected under bark on decaying spruce stump.

Adults September 1.

Randolph county.

4. Pterostichus honestus, Say.

Collected under bark on spruce log.

Adult September 1.

Randolph county.

5. Platynus angustatus, Dej.

Collected under bark on spruce stump.

Adults September 1; July 11.

Randolph and Grant counties.

6. Platynus sinuatus, Dej.

Adult September 1.

Collected under dead bark on spruce $\log$.

Randolph county.

7. Dromius quadricollis, Lec.

Adults March 30.

Collected under bark on spruce log.

Grant county. 
S. Chlænius tricolor, Dej.

Adult September 1.

Collected under bark on Spruce log.

Randolph county.

\section{Order COLEOPTERA; Family STAPHYIINID \#.}

\section{Homalota sp}

With Tomicus caelatus, Tomicus pini. Polygraphus, rufipennis in Spruce and Dendroctons frontalis in Pine.

Adults September 1, 22, 25; October 21, 22; April 14, 17; May 4, 30; July 13.

Randolph. Monongalia, Tueker, Wood and Pendleton counties.

10. Quedius peregrinus, Grav.

Adult August 29.

With Scolytids under spruce bark.

Randolph county.

11. Xantholinus cephalus, Say.

On bark of Black Spruce tree infested

Adults March 29. with Scolytids.

Tucker county.

12. Baptolinus longiceps, Fab.

With Po'jsraplus rufipennis in

Adult September 1; March 28.

Spruce bark.

Randolyh and Tucker counties.

13. Olophrum obtectum, Er.

Adult October 17.

Collected under Pine hark.

Wood county.

14. Siagonium americanum, Melsh.

With Dryocoetes autowrapluts in Spruce bark, and Dryocoetus 11. sp. Adults May 8 ; July 11 . Larvx May 8. in Birch bark. -

Tucker and Grant counties.

\section{Order COLEOPTERA; Family PHALACRID}

15. Olibrus bicolor, Gyll.

Adults in Spruce bark and on Locust leaves. Larvie in Ergot on grass. Adults March 10; July 9; August 29; May 21. Larvæ July 9. Wood. Randolph and Gilmer counties. 


\section{Order COLEOPTERA; Family CORYLOPHID E.}

16 Sacium biguttatum, Lec.

Ahults August 22.2.

I'nder bark on dead Beech.

Tyler rounty.

Order COLEOPTERA; Family COCCINELLID \&.

17. Hippodamia convergens, (iuer.

PREDACEUUS.

Attacks Aphids on Apple and Locust

Adults July 17, 20; Aurust \&, 24, 2.; September 7; Ortober 4 inay 4

Wool, Monongalia, Marshall, Brouke, Tucker and Jackson rounties.

Is Coccinella 9-notata, Hbst.

PREDACEOUS.

Attacks Aphids on Locust and Apple.

Ailults April 17, 18; June 24; July 2, 11, 17, 20, 30; October 3. WVeb-ter. Wool, Marshall, Monongalia, Randolph, Cabell and Wirt counties

19. Coccinella sanguinea, Linn.

PREDACEOUS.

Attacks Aphicls on Cherry, Locust and Apple.

Adults June 25, 26; July 7; August 7 ; September 7 ; October 4. Monongalia, Wood and Upshur counties.

20. Adalia bipunctata, Linn.

PREDACEOUS.

Attacks Aphids on Locust, Box Elder, Apple and Balm of Gilead. Iclults June 1, 11; July 16, 20; Angust 1, 7; September T. Pupex June 1. Larvic June 1.

Hancock, Cabell, Monongalia and Marshall counties.

21. Anatis 15-punctata, Oliv.

PREDACEOUS.

Attacks Aphids on Box Elder.

Adults April 27; June 11; July 21. Pupe June 11. Larve June 11 .

Ionongalia, Wood and Wetzel counties. 


\section{Order CLEOPTERA; Family ENDOIMYCHID सE.}

22. Endomychus biguttatus, Say.

Adults October 15; December 6.

With Scolytids under Pine bark.

Marion and Monongalia counties.

\section{Order COLEOPTERA; Family COLYDIID王.}

23. Coxelus guttulatus, Lec.

Under dead bark on Beech and Pine.

Adults May $6 ;$ July 22.

Hampshire and Tyler counties.

24. Aulonium parallelopipedum. Say.

Under bark on dead Beech and Black Oak.

Adults July 30 ; A ugust 22.

Tyler and Wood counties.

25. Aulonium tuberculatum, Lec

Adults July 13

With Scolytids in Pine bark.

Hampshire county.

26. Colydium lineola, Say.

PREDACEOUS.

With Platypus quadridentatus in sap woud of Black Oak; Xyloterus politus in Maple; Monarthrum mall in Jack Oak; in Scolytid mines in Pine liark.

Aúults July 13, 29, 30; A ugust 19, 27 ; September 12

Hampshire, Wayne, Tyler, Monongalia and Wood counties.

27. Sosylus costatus, Lec.

PREDACEOUS.

With Platypus quadridentatus in

Black Oak.

Adult July 29.

Wayne county.

28. Philothermus glabriculus, Ltc.

With Scolytids under Spruce bark.

Under dead Locust bark

Adults August 29; Octıber 11.

Randolph and Monongalia counties. 
Order COLEOPTERA; Family CUCUJIDAE.

29. Silvanus planatus, Germ.

Adults Mareh 18.

Inder hark on dead Yellow Locust.

Wood county.

30. Catogenus rufus, Fah.

Adults April 12.

Inder bark on dead Sugar Maple.

Monongalia county.

31. Cucujus clavipes, Fab.

Adults February 25.

Inder bark on dead White Oak.

Preston county.

32. Dendrophagus glaber, Lec.

Adults September 1.

Under bark on Spruce log.

Randolph county.

33. Brontes dubius, Fah.

Adults April 9.

Under bark on dead Locust.

Wood county.

Order COLEOPTERA; Family MYCETOPHAGID尼.

34. Litargus 6-punctatus, Saly.

Adults April 17.

Under bark on dead Beech.

Wood county.

35. Diplocoelus brunneus, Lec.

Adults August 22.

Under bark on dead Beech.

Tyler county.

\section{Order COLEOPTERA; Family HISTERID王.}

36. Hololepta fossularis, Say.

Under bark on dead and dying Populus, Hickory and Locust.

Adults April 5; June 13; August S; October 11; Eggs October 11 .

Wood county. 
37. Hister parallelus, Say.

With Dryococtes autographus in Spruce. Tomicus Cacographus and Gnathotrichus inateriarius in Pine Arlults May 18; June 24, 29; July 20; November. Monongalia, Wood and Marshall counties.

3S. Hister cylindricus, Payk.

With Tomicus cacographus and TomAdults, Nay 5,8 ; A ugust 12. icus colligraphus in Pine bark.

Wood and Pendleton counties.

39. Paromalius estriatus, Lec

Under Hickory and Black 1)ak bark. Adults March; July 30; October 27.

Wood and Monongalia counties.

40. Paromalus bistriatus, Er.

With Polygraphus rufipennis in Spruce bark.

Aclults July 11; August 30.

Randolph and Tucker counties.

41. Paromalus difficilis, Horn.

With Polygraphus rufipenuis and Dryocoetes affaber in Spruce hark.

Arults July 8 ; August 28; September 1; Pupæ July \&. Randolph county.

42. Plegaderus transversus, Say

With Tomicus cacographus in Pine bark.

Adults June 24, 29; July 20.

Wood and Marshall counties.

Order COLEOPTERA; Family NITIDULIDA®.

43. Colastus unicolor, Say.

With Pityophthorus confuis and Tom. icus calligraphus under bark on dead pine

Adults Nay 4.

Pendleton county.

44. Epuræa avara, Rand.

Adult July 8 .

Under dead bark on Spruce logs.

Grant county. 
45. Epuræa truncatella, Mann.

A clults March 26; .July.

With I'olygraphus mefpennis in Spruce bark.

'Tucker and (irant counties.

46. Prometopia 6-maculata, Say.

Arlults August 22.

Under bark on dead Beech.

Tyler county.

47. Cryptarcha ampla, Er.

Adult: Mav 1

With Cyllene rotinice larver under bark on living Yellow Locust tree

Wool county.

48. Ips fasciatus, Oliv.

With Cyllene robinice larva under hark on living Yellow Locust tree and Xjloterus lin'tlatus on Spru.e $\log$.

Arlults March: April 5; May 1,9

Wood couniy.

49. Nitidulidæ larvæ.

Ips fasciatus?

With Xylotorus bivittatus in Spruce wood; Platypus compositus in Bass wood. With Cyllene rolinuce larve in Locust bark on green tree.

Larvie May 14; June 1; July 9, 21.

Wood and Grant counties.

50. Ips sanguinolentus, Oliv.

With Nyloterus politus under Sugar Maple bark; also feerling on Black

Achults April 5; May 9. Walnut sap.

Wood and Grant counties.

51. Rhizophagus dimidiatus, Mann.

Adults March 26; September 1.

With Scolytids under Spruce bark. Randolph and Tucker counties.

52. Rhizophagus bipunctatus, Say.

Under bark on dead Beech with Monarthrum fasciatum and with Dryocoetes 11 . sp. in Birch bark.

Adults March 21; A pril 18; Octoher 21.

Wood, Pendleton and Monongalia counties. 


\section{Order COLEOPTERA; Family LATHRIDIID}

53 Latridius maculatus, Lec.

Adults October 21.

Under bark on dead Beech.

Wood county.

54. Latridius opaculus, Ler.

Adults October 21.

Under hark on dead Beech

Wood county.

55. Corticaria sp.

Adult May 21.

On Y (.llow Locust leaves.

Wood county.

56. Corticaria elongata, Hum.

With Tomicus calligraphus under

Adults July 27.

bark on Pine.

Wirt county.

\section{Order COLEOPTERA; Family TROGOSITID AE.}

57. Nemosoma cylindricum, Lec.

With Tomicus cacographus in Pine bark.

Adults May 20.

Wood county.

58. Trogosita virescens, Fab.

Under bark on dead and dying White and Yellow Pine trees.

Adults May 4 ; July 20.

Marshall and Hampshire counties.

59. Tenebrioides corticalis, Melsh

Under bark on dead Apple tree, and with Phloeotribus frontalis in bark of live Mulberry.

Adults September 7. Pupæ April 25. Adult emerged May 12. Monongalia and Wood counties.

60. Tenebrioides castanea, Melsh.

Adults August 22.

Under bark on dead Beech.

Tyler county. 
61. Bactridium ephippigerum, (iuer.

Under partly green bark on Basswood log, and under bark on dead Becch

Adult April 17; July 21.

IVetzel and Wood counties.

\section{Order COLEOPTERA; Family ELATERID}

62. Tharops ruficornis, Say.

WOOD BORER.

In dead Beech wood and on bark of

Adults May 27; June 16; July 10. dead maple.

Grant, Randolph and Monongalia counties.

63. Alaus oculatus, Linn.

WUOD BORER.

In green Oak wood and in decaying wood.

Adults June 19. Larvie May 8; March 10.

Wood county.

64. Hemirhipus fascicularis, Fab.

PHEDACEOUS.

Larva attacks Cyllene picta larvæ in Mulberry wood.

Adults bred June 5; Larvæ April 9.

Wood county.

\section{Order COLEOPTERA; Family BUPRESTID AE.}

6.5. Chalcophora campestris, Say.

BARK BORER.

Said to infest dead and dry Syca-

Aduits flying May 18; June 10. more, (Packard).

66. Dicerca divaricata, Say.

BABK BORER.

Attacks bark on dead Peach tree.

Collected Adults May 11; July 12; September 12.

Wood and Randolph counties.

67. Dicerca obscura, Fitch.

BARK BORER.

Adult May 18.

On dead Oak branch.

Wood county. 
68. Dicerca lurida, Fal.

Adult flying May 2.

BARK AND WUOD BURER.

Said to attack Pig Nut Hickory (Harris).

Wood county.

69. Buprestis rufipes, Oliv.

Adult flying July 20, August 19.

Monongalia county.

70. Buprestis lineata, Fah.

Adult ftying June 21.

Wood county.

71. Melanophila fulvoguttata, Harv.

B.IRK BORER.

Infests green bark on living, injured

Adults March 31; May 9: June 16; July 17.

and dying Hemlock trees.

Upshur and Grant counties.

72. Melanophila sp. a

BARK BOKER.

Adult June 21.

Collected on Spruce logs.

Randolph county.

73. Anthaxia æneogaster, Lap.

BARK BuKEK.

Infests bark on dying Hickory trees.

Adults A pril 23; May 21.

IVood county.

74. Anthaxia viridicornis, Say.

BARK BURER.

-Infests lying Willow an 1 Spruce?

May 4; June 25; August 30; September 11.

Wood and Randolph counties.

75. Anthaxia flavimana, Gory.

TWIG BORER.

Adult May.

Infests dying Plum branches.

Wood county. 
76 Chrysobothris femorata, Fab.

IAARK AND WO(H) BORER.

Infests hark on logs, stumıs, injured and dying trees; White Oak, Black ()ak, Apple, Hickory, Maple, Basswood

Ailults May 1, 15, 15, 20; June 16, 23; July 20, 27. Larve Manch 20 and Norember to April.

IVond, Grant, Wetzel, Upshur and Monongalia counties.

77. Chrysobothris pusilla, Lap. and Gory.

BARK BUREK,

Adults September 1.

Collected on Spruce logs.

Randolph county.

78. Chrysobothris azurea, Lec.

BARK AND WOOD BORER.

Infests Pine Bark and wood on

Adults May 3, 5, 15. branches of dead trees

Wood, Hampshire and Grant counties.

79 Acmæodera ornata, Fab.

Adults fiying June 16,24 .

IVood county.

S0. Acmæodera culta, Wéb.

WOON BORER.

Infests bark and wood of dead Hickory. Adults found on Locust leares,

Dandelion and Wild Rose Blossoms.

Adults May 18, 21; June 23; September 15 .

I'ood and Monongalia counties.

S1. Buprestid sp, c.

Adult July 24.

On lead Hickory.

Ionongalia county.

S2. Agrilus otiosus, Say

BARK B.RER.

Infests bark on dead twigs and branches of Hickory, Black Walnut. Arluils feed on Walnut and Hickory leaves.

Aclults April 14, 23; May 15, 18, 21, 30; July 4, 16, 2.5.

Wood, Upshur, Calhoun and Monongalia counties. 
83. Agrilus bilineatus, Web.

BARK BORER.

Infests green bark on stumps, living, injured and dying trees. May cause the death of trees. Infests White Oak, Black Oak and Chestnut.

84. Agrilus fallax, Say.

\section{BARK BORER.}

Infests bark and wood of dying branches on living and dying Hack-

Adults May 2. berry.

Wood county.

85. Agrilus politus, Say.

BAHK BORER.

Infests green bark on living Willow trees. May be the primary cause of death of young trees

Adults June 13, 27. IPupe May 4; June 27 Larvæ May 5, 20. Grant and Wood counties.

86. Agrilus egenus, Gory.

BARK BURER.

Larvæ infests bark on dead and dying branches on living and dying Yellow Locust trees; adults feeding on leaves, common.

Adults April 24; May 15, 18, 20, 21, 30; June 1, 13, 15, 16, 27; July 16. Pupæ May 20; Larvæ May 20.

Monongalia, Wond and Hancock counties.

57. Agrilus sp, b.

BABK BORER.

Infests green bark on White Oak

Adults April 29; June 25. stumps.

Wood county.

88. Agrilus sp. c.

BARK BWREK.

Infests green bark on living and

Adult April 14 dead Dogwood.

Wood county. 


\section{Order COLEOPTERA: Family LAMPYRID}

s.). Lucidata atra, Fab.

Under dearl bark on Spruer and Apple trees.

Arlults March 29; June 16; November 2.

Wood, Monongalia and Tucker counties.

(). Ellychnia corrusca, Linn.

Adult March 29.

Common on Spruce bark.

'Tucker county.

91. Chauliognathus pennsylvanicus. De G.

PREDACEOUS.

Larrat attacks Ecdlyolopha insiticina (Locust Twig' Borer) in Yellow

Larvie September 17. Locust twig.

Monongalia county.

\section{Order COLEOPTERA; Family MALACHID王.}

92. Attalus scincetus. Say.

With Scolytus rugu'osus in Apple lark

Adult March 21.

Monongalia county.

\section{Order COLEOPTERA; Family CLERID王.}

93. Elasmocerus terminatus, Say.

PKED: CEOUS.

With Sinoxylon basilare, Agrilus otiosus and Chramesus ic, rim in dead Hickory branches.

Arlults Hying June 22. Adults bred from Hickory April 23. Wood and Monongalia counties.

94. Cymatodera bicolor, Say.

\section{PREDACEULS}

With Phlo'osimus dentatus! in Cedar bark.

Adult May 15.

Woorl county. 
95 Cymatodera inornata, Say.

PREDACEOUS.

In outer bark on grem Pine. Proba-

Adult October 15 .

bly attacks Hymenopterous insects

Marion county.

96. Clerus quadrisignatus, Say. var. nigripes, Say.

PKEUACEOUS.

With Tomicus cacographus and Pityophthorus pullus in Pine bark and with Phlocosinus dentatus in Red Cedar bark.

Aduit May 4, 9

Graut county.

97. Clerus sp. a.

PREDACEOUS.

In pith of dead Sumach. Probably attacks Adyucrus su

Adults bred April 20; June 25. Larvæ April 1.

Wood county.

98. Thanasimus trifasciatus, Say.

PREDACEOUS.

With Polly graphlus rufipennis under Spruce birk.

Adult March 28; August 29. Larva March 28.

Raudolph and Tucker counties.

99. Thanasimus dubius, Fab.

PREDACEOUS.

Attacks Tomicus cacographus, Tomi ius calligraphus Dendroctonus frontalis, Pityophthorns pullus, Pityophthorus tuburculatus, Pityophthorus sp. d. in Pine bark; Polygrafhus rufipen,is in Spruce bark and Hylesiuus opaculus in Elm bark.

Arlults May 2, 8, 30; August; September 1; Outober 6; November; Larve March 28, 311; May 23; July 21, 30; August 29 ; September 7 , October 6, 10, 21.

Randoph, 'Tucker, Hampshire, Monongalia, Grant and Wood counties. 
100 Clerus formicarius, $\mathrm{L}$.

Thantstunts: formucarius, I.

The Luropean Bark Beetle Destroyer. Destructire to Scolytirls in European coniferous forests (one thousand live specimens of alults, larvie and pupa imported to America in October, 1892 , by the West Virginia Experiment Station, ai led by owners of ine forests. First adults collected by me in Government forest of Pinus sylvestris near Hagenau, Elsace, Germany, dugust 29th, 1892. First adults set free in America, in Pine forests near Morgantown, West V'irginia, on October 10th, 1892 .

101. Thaneroclerus sanguineus, Say

PREDACEOUS.

On Hemlock stump with Xyloteris

Adult May 8 . hrittatus.

Grant county.

102. Phyllobæuus dislocatus, Say.

PREDACEOUS.

Attacks Polygraplutes rufipenn's in Black sipruce and Potjophthorus consimulis in Sumach (Rhus glibra) and with Scoly'us rugulosus in Apple bark.

Adults bred June 16; 20, 22; December 7 .

Riandolph and Monongalia counties.

103. Chariessa pilosa, Forst.

PREDACEUU.

Under bark on dead $W$ almut and in read grape vine.

Alults April 15; May.

lot Enoplium quadripunctatum, say.

PREDACENS

Bred from dearl Black $\mathbb{I V}^{r}$ almut branches.

Arinlt April s.

Wood county 
105. Orthopleura damicornis, Fab.

l'REDACEOUS.

Adults July 27. ory wood.

Wood county.

106. Clerid larvæ, sp. a.

PREDACEへUS.

With Dendroctonus frontalis, Tomicus arulsus and Tomicus cacographus larva in Pine bark.

Larvæ June 23, 29 ; October 14.

Wood county.

107. Clerid larvæ, sp. b.

PREDACEOUS

With Polygraphus rufipennis, larve in

Larvæ July 23; March 26. Spruce.

Tucker and Wetzel counties.

108. Clerid larvæe, sp. c.

Larvæ March 2; July 13.

With Phlo'otritus liminaris in Wild Cherry and Scolytus rugulosus in Apple bark.

Monongalia county.

109. Clerid larvæ, sp. d.

PREDACEOUS.

With Scolytus rugulosus in Apple bark; Scolytus quadrispinosus in Hickory bark and Scolytus muticus in Hackberry bark.

Larvæ March 2; July 20; October 7; November 23. Marshall and Monongalia counties.

110 Clerid larvæ, sp. e.

Larvæ July 20.

Marshall county.

111. Clerid larvæ, sp. f.

Witli Tomicus Pini in Pine bark.

Larva March 2.

Monongalia county.

With Cerambycid larva in Black Walnut bark. 


\title{
Order COLEOPTERA; Family PTINIDÆ.
}

112. Xestobium squalidum, I.e.

\author{
WOOI) BOHER.
}

Irfests dead wood of Black Spruce. Arlults bred. Larva collected March 31; May 9. Giant county.

113. Ptilinus ruficornis, Say

$$
\text { WOOD I3ORER, }
$$

Infests dead and dry wood where the bark has been removed on living trees, $\operatorname{logs}$ and stumps of Beech, Wild Cherry, Naple, Cultivated Cherry, Iron wood.

Adults Feb. 17; April 12; June 10, 25, 27; July 10, 17. II ood, Monongalia and Grant crunties.

114. Sinoxylon basilare, Say.

Infests wood of dead Hickory and Mulberry.

Adults April 14, 23; May 4, 15, 22. Larve May 4; March 18. Wood county.

11j. Bostrychus bicornis, Web

WHOD BORER

Infests dead wood of Beech, Elm? Apple?

Adults July 16, 30; Aug. 20.

Hancock, Wayne, Monongalia and Wood counties.

116. Lyctus striatus, Melsh.

"POWDER POST" BEETLE.

WOOD BORER.

Infests seasoned wood of Hickory, Yellow Locust, Wild Lherry. Tery injurious to stored handles, spokes, hoops, \&c.

Adults bred Feb. 17, 29; June 23; July 7 . Larve Jan. 1, 9. Grant, Monongalia, Pocahontas and MLarshall counties. 
Order COLEOPTERA; Family LYMEXYLID王.

117. Hylecœtus? larva, sp a.

OAK TLUBER WURM.

Infests wood of White Oak Logs and trees. Cause "pin holes" in heartwood and sap wood Very injurious

Larva, Mar. 15.

Wood county.

118. Lymexylid larva, sp a.

WOOD BOREK.

CHESTNUT TIMBER WuRM.

Infests wood of living, injured and dead Chestnut trees and logs. Causes "pin holes" in wood.

Larve May 20; July 29.

Wood county.

119. Lymexylon, sp. a.

W(OOI) BORER.

Infests wood of dying Hemlock trees.

Adult bred. Larve April 1.

Marion county.

120. Lymexylon? larvæ sp. b.

WMUD BURER.

Infests wood of Magnolia logs. Causes "pin holes" in wood.

Larve July 21.

Narshall county.

121. Lymexylon(?) sp. c.

WVOCD BURER.

Larvat bore through the outer sap wood just beneath the surface and directly across the grain of the wood of large Aspen.

Adults bred April 1. Pupe March 27; Larve August 10; November 7 ; Narch 27

Monongalia county.

Order COLEOPTERA; Family SCARAB\#IDAE.

122. Clœotus aphodioides, Ill.

Under bark on dead Chestnut Oak tree,

Adults July 30.

IVood county 
12:) Clœotus globosus. Say.

Aclults muly $\$ 0$.

Under bark on dead Black Oak.

Wood county.

124. Macrodactylus subspinosus, Fal.

R(ISE BEETLE.

FUIAAGE FEEDER.

Infest: Yellow Locust ambl nudny

Alults Nay 30 ; June 12, 16, 23.

other fore-t and shate trees.

11 oml and Honongalia counties.

1.5. Lachnosterna fusca, Froh.

MAY IBEETI,E.

FOLLACE FEEDER.

Adults infest Oaks, Persimmon and many other forest and shade trees, oceasionally defoliating large treos.

Larve feecl on roots of grass, ife. Common throughout the State.

1ㄴ) Lachnosterna hirticula, Knoch.

WAY BEETLE.

FOLIAGE FEEUER

Infests Oaks anci other forest and shade trees. Defoliates large trees.

Adults in company with above species dofoliating Oalk forest in Greenbrier county June 15, as reported hy Erlitor of "Greenbrier Independent."

127. Valgus canaliculatus, Fab.

Infests decaying wood of Pine and

Adult F'eb. Peach stumps.

Wood county.

Order COLEOPTERA; Family SPONDYLID王.

128 Parandra brunnea, Fał

Adults .July 30.

Under bark on dead Beech.

Wayne county. 


\section{Order COLEOPTERA; Family CERAMBYCID无.}

129. Prionus laticollis, Drury.

WOOD BORER .

Infests dead wood of Pine stumps, Roots of living Black Oak and woud of Oak logs?. Probably a destructive species.

Adults July 20; A ug; Pujwe June 20; Larræ? June; Eggs July. Upshur, Monongalia and Wood counties.

130. Asemum moestum, Hald.

WOOD BOKER

Inferts wood near base of stumps and dying Pine and Spruce trees. Hastens death of trees and decay of wood.

Adults April 20; May 8; June 21. Pupa April 20; Larvæ A pril 20; September 2.

Wood and Raudolph counties.

131. Tetropium cinnamopterum, Kirby.

BARK AND WUOI) BuRER.

Infests green bark and wood on injured and dying Black Spruce trees. Very injurious. Hastens death of trees and causes rapid decay of wood.

Adults March 31; Julv 9. Larve March 29; 31; July 7.

Grant and Tucker counties.

132. Smodicum cusujiforme, Say.

BARK BORER.

Under bark of dead Honey Locust, Beech and $\mathrm{E} \mathrm{m}$.

Adults .June $25 ;$ July $16,20$.

Wood, Wetzel and Hancock counties.

133. Phymatodes variabilis, Fab

THE WHITE OAK PIYMATODES BARK AND WOOD BORER.

Infests White Oak, (Packard.)

Adults Hying May 9, 21; June 15, 16.

Wood and Harrison counties.

134. Phymatodes dimidiatus, Kirby.

BARK BORER

In bark of Spruce log.

Adults June 21.23. Larvæ June 12.

Monongalia, Pendleton and Randolph counties. 
135. Callidium antennatum, Newm.

W(HO1) BORER.

Intests wood of dying and dead Pine trees. Canses rapid decay of wored. Adults collected May 3 . Also bred later. Larve May 3 to ti. Hampsire county.

136 Chion cinctus, Drury.

WOIII) BORER.

Infests deal branches of Black ()ak

Adults June 16; Larra March 18; December 20).

IVood county.

137. Eburia quadrigeminata, Say.

W(U)1) B()KER

()1 dead Beech and Ehm. Prohahly

Adults July 17, 20, 22. infests these trees.

Wetzel, Tyler and Brooke counties

138. Elaphidion mucronatum. Fab.

IVIMD BORER.

Infest:- deat lark and wuod of

Beech Green wood of living Sugar

Milple and bark of Black Oak.

Adults collected July 14, 25; August 1, 19. Bred Larve Novemiber 26. Adults emerged later.

Wnod, Webster, Cahell and Monongalia counties.

139. Elaphidion parallelum, Nıwm.

OAK PRUNER.

'I W'IG BORER.

Infests small, living branches of Black Oak, Jack Oak and $\mathrm{W}$ alnut.

Causes the branches to break and fall

Adults January; February 2; March 13. Larve July 29; August 6.

Woorl and Monongalia counties.

140. Molorchus bimaculatus, Say.

WOOD BOHER

Infests dead twigs on living Black

Walnut trees and dyin? wood of Dogivood.

A dults bred April 8, 14; Larva A pril 14.

Wood county. 
141. Purpuricenus humeralis, Fab.

Adults collected June 23.

Collected on Oak leaves.

Wood county

142. Batyle ignicollis, Say.

Arlults collected July 15 .

IVood county.

143. Cyllene pictus, Drury.

WOOW HWRER.

Infests dead hranches and small trees,

Mu'berry and Ilickory.

Adults April 9, 14; Pupe April 9. Lall væ April 9.

Wrod crunty.

144. Cyllene robiniæ, Forst.

THE L.OCUST BORER.

Infests live bark and wool of healthy Yellow Locust trees. Very injurious and lestructive. The primary cause of leath of trees.

Arults August 25; Sept 16, 17. Larve May 1, 20.

Monongalia Mineral and Wood counties.

145. Arhopalus fulminans, Fab.

WOOD BURER.

Infests sap wood of Oak, Chestnut and Hemlock logs Causes large worm holes in outer wood. Hastens decay.

Adults April 18; May 12, 20; June 16; July 5, 24; Aug. 10.

Pupae April 18. Larvae 18. Eggs Aug. 10.

Wood, Calhoun and Monongalia counties.

Xylotrechus colonus, Fab

WO(H) BORER.

Infests bark and wood of logs and dear trees of Black Oak, White Oak, Hickory, Chestnut, Ash, Elm. Hastens decay of wood.

Adults May 8, 11, 15, 20; June 25; July 3. Pupae April 23; Larvae April 12, 1 x.

Wood, Monongalia and Calhoun counties.

147. Neoclytus luscus, Fab.

BAKK AND WOOD BORER.

Attacks dead Ash.

Aclult Aug. 10. Eggs? Aug. 10.

Monongal.a county. 
11. Neoclytus capræa, Sily

$$
\begin{aligned}
& \text { lufests saw logs and lying trees of } \\
& \text { black 1:h. Fory injurious to salw } \\
& \text { logs, boring numerous holes through } \\
& \text { the wool }
\end{aligned}
$$

dilults emerging March 19); May :30. l’upar March 20. Larval March 19, 20, 24.

Irood comity.

11!). Neoclytus erythrocephalus, Fil.

$$
\text { WOCD BHRE }
$$

Mining in bark and salp wood of dying and dearl trees. Iufests Laeust, llickory, Oak, P'erimmon, Matphe, Eln, Willow, Puach Apule.

Aclu'ts Maty 1, 15, 19, 20, 21; July 14, 24; August 4 ; September 2 I'upar Manch 18; M:1y 1 .

IVood, Monongalia, T'ucker, Randolph and Kanawha counties.

150. Clytanthus ruricola, Olir.

Arults April 12; June 15.

$$
\text { Infests dead Sugar Maple. }
$$

Harrison county.

151. Distenia undata, Oliv.

BABK AND WU(O) BORER

Collected adult under loose bark on

Adult July 25.

deat Chertnut.

Wood county.

15. Rhagium lineatum, Oliv

BARK BORER.

Mining under bark of dying trees. Changing to beetle in ship, cocoon under bark. Infests pines.

Adults April S; May 5, 9 ; Oct. 17; Dec. 19. I'upa Oet. Larvil. July 1.4 .

Comnon in pine forests.

153 Monohammus scutellatus, Say.

WUOI) BOREK

WHITE PINE "SAWYER,"

Boring in sap and heart wood of saw logs, dying and dearl trees. C'anse large holes in wood. Hastens decay. Very injurious to timber. Infests Whitc Pine.

Aclults May 5; Larvie June 23; Oct 10.

Pendleton and Marion counties. 
154 Monohammus confusor, Kirby.

WOOI) BORER.

SPRUCE SAWYER.

Boring large holes in saw logs and

Adults Aug. 29.

dying trees. Infests Black Sipruce

Randolph county.

155. Monohammus marmorator, Kirby.

WOOI BORER.

PINE SAWYER.

Boring large $r$ oles in saw logs, dying and dearl trees. Infests Yellow,

Adults July 12, 24. Pjtclı and Scrub Pines.

Monongalia county,

156. Dorcaschema nigrum, Say.

Adult June 23

Flying in Spruce forest.

Pendleton county.

157. Goes oculatus, Lec.

Adult collected flying July 14 .

Randolph county.

15S. Leptostylus aculiferus, Say.

BAHK AND WUOD BORER.

Infests dead and dying Apple and

Adults A pril 19; June 1:3, 18.

Maple trees.

Monongalia and Woot counties.

154. Liopus variegatus, Hald.

Adult collected flying Oct. 3 .

Monongalia county.

160 Liopus alpha, Say.

BARK AND WOOI BORER.

Infests Yellow Locust twigs on dead tree.

Adults bred April 24. Collected June 10.

Monongalia county.

161. Liopus punctatus, Lec.

WOOD BURER.

Infests dying and dead Plum and Dogwood.

Adults bred May 11, 20. Pupæ April 25; May 4.

Wood county, 
162. Lepturges signatus, Lar.

BARK ANI) WUWD BORER.

Infests dead Beoch branches.

Adults bred May. Larvae April 12.

Monongalia county.

163. Hyperplatys aspersus, Sily.

BARK ANI) WOOB) BOREK.

Infests dying and deal Sumach,

Adults bred April 23 ; May 2.

Hackberry and White Walnut.

Wood county.

164. Hyperplatys masculatus, Hald

Collected on dead Maple and Yellow Locust branches.

Adults May 26; June 13.

Monongalia county.

165. Urographis fasciatus, De G.

BARK BORER.

Infests dying and deard Chestnut. Black Oak.

Adults June 11; July 24. Pupre Sept. 12

Wood and Monongalia counties.

166. Graphi surus pusillus, Kirby.

Adults August 29; Sept. 2.

Infests bark on dead Black Spruce.

Randolph county.

167. Ancanthocinus obsoletus, Oliv.

Collected on Spruce stump. Bred from Pine bark

Adults collected July 9. Larva Nov 7.

Grant county.

168. Hoplosia nubila, Lec.

Adult May 15.

BARK BORER.

Infests bark on dead White Elm.

Wood county.

169. Pogonocherus penicellatus, Lec

WOOD BOLER,

Infests sap wood of dying and dead

Adult July 16. Sumach.

Hancock county. 
179. Eupogonius vestitus, Say BARK A.NO TWI BORER.

Adults bred May 30.

IIonongalia county.

171. Oncideres cingulata, Say

TILE IICKORY TWIG GIRDI.ER.

Girdles the twigs and small branches on living trees. Larva develops in deal portion Infests Hickory, Basswond.

Adults July 22; October 29. I arva April 12.

Marion, Wood and Mason counties

172 Saperda discoides, Fab.

BARK BORER.

Infests bark on dead and dying Hickory.

Adults bred May 12 and 30. Pupa April 30

Wood and Monongalia counties.

173. Oberea tripunctata, Swed. var. Myops, Hald.

Adult collected flying July 15, 20.

Randolph and Webster counties.

174. Oberea ruficollis, Fab.

Adult collected on Blackberry fruit July 25.

Wood county.

175. Cerambycid, sp. a

BARK ANU WOOD BOKER

Infests bark and wood of dying Red Cerlar.

Adults bred May 8

Hampshire county.

Order COLEOPTERA; Family CHRYSOMELIDZA.

176 Anomoæ laticlavia, Forst

FWLIAGE FEEDER

Collected on Yrullow Locust leaves.

Common also on Sumach leaves.

Adults June 10, 17 ; July 5.

Monongalia, Wood and Gilmer counties. 
177 Chlamys plicata, Fib

HOL.1AGE FEEIEH.

Larrate and adultif frepuent on Sycatmore leaves.

Adults April 17; May !1. Bred August 20. Larva $\Lambda$ ugust 2. Wood and Kanawha counties.

1is Cryptocephalus mammifer; $*$

POIAAGE WEEDER.

Common on Yellow Locust leaves. Also collected on Blackbery and Adult. May 20, 21, 30 ; June 1, 13 Raspbery loaves

Monongalia and ITood counties.

179. Pachybrachys atomarius, Melsh.

F(),AAGE FEEDER.

Frequent on Yellow Locust leaves. Adults June 16, 23, 26; July 16 .

Wood, Upshur and Hancock counties.

150. Glyptoscelis pubescens, Fab.

Adults May 2.

Collected on Pine leaves.

Wood county.

1S1. Myochrous denticollis, Say.

Adult April 19; May 5.

Collected on Yallow Locust leares

Wood county.

182 Paria aterroima, Oliv. HOILAGE FEEUER

Collected on Yellow Locust leaves. Common.

Adults May 18; June 10.

Wood county.

183. Colaspis brunnea, Fab.

FULAACIF FEEUER.

Collecterl on Yrellow Losust leares June 27 Common on Grape leaves.

Adults June 25, 27; July $16,2 \cdot 2$.

Doing comsiderable damage.

Monongalia, Wood, Tyler and Hancock countios.

*Determined hy Dr. Horn. 
184. Colaspis tristis, Oliv.

Adults June 16, 17; Aug. 24.

Collected on Yellow Locust and Blackberry leaves.

Wood county.

185. Colaspis puncticollis, Say.

Collected on Yellow Locust and Su-

Adult .Tune 1, 13; July 3, 30. mach leaves.

Wood, Wayne Monongalia and Wirt counties.

186 Doryphora clivicollis, Kirby.

Adults June 21.

Collected on Yellow Locust leaves.

Mono" gal a and Woorl counties.

187 Chrysomela saturalis, Fab.

FOLIAGE FEEDER,

Collected on Yellow Locust leaves.

Adults April 5, 16, 17; Aug. 8 .

Monongalia and Wood counties.

188. Chrysomela scalaris, Lec.

Collected on Mountain Ash leaf.

Adults June 23.

Randolph county.

189 Chrysomela bigsbyana, Kirby.

Adults July $16,30$.

HOLIAGE FEEDER.

Common on Willow leaves.

Wood and Upshur counties

190. Gastroidea cyanea, Melsh.

FOLIAGE FEHDER.

Adult May and June.

Collected on Willow leaves.

Wood county.

191. Lina lapponica, Linn.

FULIAGE FEEUER.

Common on Willow leaves.

Adults April 12, 17, 29; May 2, 4; June 16. Eggs May. Wood and Upshur counties.

192. Phyllodecta vulgatissima, Linn.

FOLIAGE FEEDER.

Very common on Black Willow leaves; eating surface of leaves

Adults, Larve and Pupæ June 16; Aug 27

Randolph county. 
193. Cerotoma caminea, Fab.

Collecterl freding on sap flowing from wounds in green bark marle by Cyllena robinice larvate on Yollow Ijocust.

Adults May 1; .June

Woorl county.

194. Phyllechthrus gentilis, Lec.

HOI,IAGE FLEDER.

Very common on Y'ellow Locust leaves.

Adults May 21, 30; June 1, 12, 13.

Monongalia and Wood counties.

19.). Adimonia cavicollis, Lec.

FOLIAGE FEEDER.

('ommon on W'ild Red Cherry leaves.

Adults common .July 10.

Defoliating trees.

Tucker county.

196. Blepharida rhois, Forst.

FOLIAGE FEEDER.
Collected on Sumach.

Adults April 11, 18 .

Wood county.

197. Crepidodera rufipes, Linn.

FOLIAGE FEEDER.

Common on Yellow Locust leaves. Adults A pril 30; May 11, 18, 21, 24, 30; June 13, 16.

Wood, Upshur, Monongalia and Tucker counties.

195. Crepidodera Helxines, Linn.

FOLIAGE FEEDER.

Common on Willow and Wild Red

Adults May 23; July 10.

Cherry leaves.

Wood and Tucker counties.

199. Orthaltica copalina, Fab.

FOLIAGE FE'HER.

Common on Sumach 'eares Collecterl on Yrellow Locust leaves.

Adults June 10; July 14.

Monongalia and Randolph counties. 
200. Odontota dorsalis, Thumh.

AHULT FOLIAGE FEEDER.

LARV LE LEAF MINER.

Causes Yellow Locust leaves to turn liruwn as if scorched by fire Beetles feed on surface of leaves. Larva mine beneath surface of leaves and form blisters. Aclults also ob served fueding on Beech, Aplle, Wild Cherry and Wisteria leaves. Very common.

Adults May 11, 18, 30; June 10, 12, 15, 16, 21, 22, 26, 27, 29 ; July 16, 20; Aug. 16 ; Sept. 14, 17 ; Oct 3. Pupa June 26 ; July 6, 18 . Larve June 4, 13, 29; July 4, 6 ; Aug. 8. Monongalia, Wood, Hancock, Harrison, Upshur, Tyler, Preston. and Tucker counties.

201. Odontota nervosa, Panz.

\section{FOLIAGE FEEDER}

Very common on Yellow Locust leaves; also feeds on Apple leaves. Adults April 30; May 18, 21, 30; June 10, 13, 26, 27; July 16. Monongalia, Wood, Hancock and Jackson counties.

202. Coptocycla guttata, Oliv.

Adults May 18 .

Collected on Yellow Locust leaves.

Wood county.

\section{Order COLEOPTERA; Family TENEBRIONID Æ}

203. Centronopus calcaratus, *

Adults June 16.

Collected on Hemlock log.

Upshur county.

204. Hypophloeus cavus, Lec.

Adult July 2:.

With Xyleborus celsus in Hickory.

Tyler county.

*Determineā ly Dr. Riley. 
20.). Hypophloeus parallelus. Melsh.

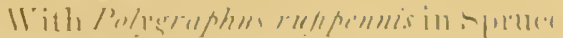

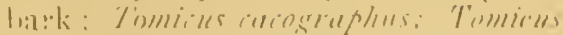
prini: Dendrestomus fromtulis: (')rpturgus pusilus in l'ine hark ant C\%ra-

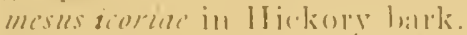

dilults . M:

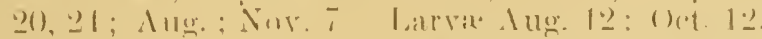

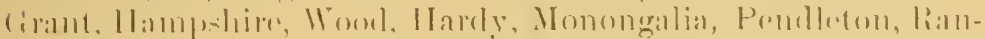

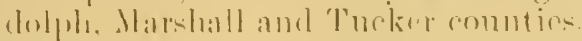

2ni. Hypophloeus thoracicus, Melsh.

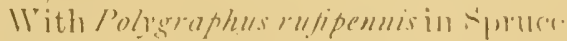
bark.

Adult Aug. 2!!

liamblph rounty.

2(1). Hypophloeus bicolor, Melsh

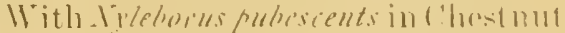
wood and linelueres juscolus in lilareli ()ak wood.

Adult May 20: Iuly:30.

IVool rounty.

20). Hypophloeus sp. a.

Ailult July 30.

Woorl county.

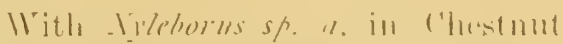
():ik woril.

2()؛. Tenebrionid lırvæ.

With f'olveraplus rufipemmis in sipure birk.

Larvi" August 30.

Randolph county.

\section{Order COLEOPTERA; Family MELANDRYÍA}

2)10. Xylita lævigata, $[[\mu] w$.

WIMI) B(HKRT:

Arlults March 2!

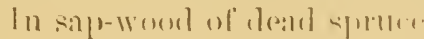

'Tucker county.

211. Serropalpus barbatus, Sh(1:1l.

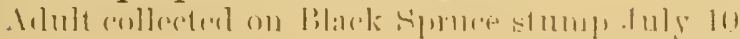

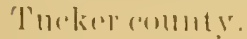


Order COLEOPTERA; Family PYTHIDAE.

212. Pytho niger, Kirby.

BARK BORER.

Infests bark on dying and dead Adults in chip cocoons April 1; May 9.

White Pine and Black Spruce.

Marion and Grant counties.

213. Pytho americanus, Kirby.

BARK BOREK

Infests dying and dead bark on Pine

Adults February 20.

logs and stumps. Common.

Wood county.

\section{Order COLEOPTERA; Family MORDELLID A®.}

214. Tomaxia bidentata, Say.

Collected on bark of dying Hickory tree.

Adult June 29.

Wood county.

\section{Order COLEOPTERA; Family ATTLEABID无.}

215. Attelabus nigripes, Lec.

Collected on Sumach and Yellow Locust leaves.

Adults June 23, 27; July 3; August 16.

Wood, Wirt and Monongalia counties.

216. Sitones flavescens, Marsh.

Adults June 27.

On Lncust leaves.

Wood county.

Order COLEOPTERA; Family CURCULIONIDÆ.

217. Apion nigrum, Hbst.

FOLIAGE FEEDER.

Very common, feeding on tender Yellow Locust leaves.

Adults May 18, 21, 25, 30; June 10. 12, 13, 16, 24, 27 ; July $16,22,30$; September 10

Wood, Monongalia, Hancock, Jackson, Tyler and Wayne counties. 
218 Listronotus latiusculus, Boh.

Under Reed Cedar bark.

Adults Narreh 10; April 1̄; May 21.

Wood cuunty.

219. Pissodes strobi, I'cek.

TWITH AND BSARK BORER.

WHTE PINE WEEYL.

Attacks the terminal $t$ wigs on small living trees, causing dwarted and deformed trees, also infests bark on logs, and trunks of living and dying Pine and Spruce.

Alults Iune 27 ; July 8, 14, 24; Aug. 4; Oct 25. Pupae June 23, 29; July 8, 13; Larve June 2.9; July 8, 11; Oct. 25; Nor. 30.

Randolph, Grant, Pendleton, Pocahontas, Hardy, Monongalia, Wood, Grant, Mineral and Hampshire counties.

\section{Hylobius abietes.*}

\section{BARK BORER.}

Adults Apr l 1:1; May 18; July 14.

Collected on dying Pines.

Wood, Hampshire and Monongalia counties.

221. Otidocephalus chervolatii, Horn.

Adult July 16

Collected on Yellow Locust leaves.

Hancock county.

222. Magdalis olyra, Hbst.

BARK BORER.

Common under and in bark on dead Hickory.

Adults bred April 14; May 12, 15. Pupæ April 23.

Wood county.

223 Magdalis inconspicua, Horn.

TWIG BORER.

Adults May 13.

Infests dead twigs on Black Walnut.

Wood county.

BARK BORER

Adults bred Oct. 15.

Infests twigs on dead Hickory.

Marion county.

*Determined by Dr. C. V. Riley. 
2.2. Anthonomus nebulosus, le:

Inforts Thorn linits.

Adults .J une 16 .

I'reston county.

2.25 . Orchestes niger. Horn.

Adults fuly 30 .

Collected on Willow leaves.

II'ayne county.

226. Orchestes ephippiatus, sily.

Adult July 30

Collecterl on It illow laver.

Wayne rounty.

2.27. Gymnetron teter, Fab.

Aclults dune 24.

Collecterl on Yellow Locust leaver.

Wood and Yamison counties.

ㄴ.2. Cryptorhynchus obliquus, Say.

SUMACH RoOT BoRER.

Infests hase and roots of sumach.

Causes the death of the shrub

Beneficial where sumach "sprouts" are a pest.

Adults hed fan. 2; July 13 . Tarve July 29; Oct. 15. Marion and Wood counties.

229. Cryptorhynchus ferratus, Siy.

I)ARK BORER.

Infests bark on dead Salssafras.

Adults June 27. Pupa June 27. Larvie June 27.

Wood county

30 Copturus binotatus, i.ee.

B.IRK BORER.

Infests bark on dying sumald. Common.

Idults July 16; October.

Hancock, Marion and Monongalia counties.

2:1. Balaninus caryatrypes, Bolı

"UESTNU'T NUT WEEVIR.

Larva November 13.

Common in Chestruts.

Ifonomgalia (o)unty. 


\section{Order COLEOPTERA; Family BRENTHID西.}

23:. Eupsalis minuta, 1)rury.

ISARK LORER

Adults collereded under loose hark on deatd Blatk Oalk. Beech, Elm.

Adults April ; July 16, 22.

Wood, llancock and Ty]er counties.

Order COLEOPTERA; Family CALANDRID无.

2:3: Himatium conicum, Lec

13ARK BHLER.

Common in bark on logs and dead trees.

Arlults Mareh 26, 28; April 17; May 4; September 1 .

Randolph, Tucker, $W^{\top}$ ood and Pendleton counties

234. Cossonus platalea, Sily.

Conder dead hark on White Walnut $\log$.

Adults (dead) April 12.

Monongalia county.

23\%. Cossonus corticola, Say

Very common under dead bark on Pine and Spruce trees.

Adults April 1; .June 24; July 13, 20, 24, 27; Aug. 12, Oct. 10. Monongalia, Marion, Marshall, Wood and Hampshire counties.

\section{Order COLYOPTERA; Family SCOLYTID}

236. Platypus quadridentatus, Oliv.

TIMBER BEETLE.

Infests Black Oak and Chestnut.

237. Platypus compositus, Say.

TIJI3ER BEETLE.

Infests Black Oak, Sugar Mraple, Chestnut, Basswood, Magnolia, Red Elm, Beech and Wild Cherry.

2:38. Corthylus punctatissimus, Zimm.

TINBER BEETLE.

Infests Sassafras.

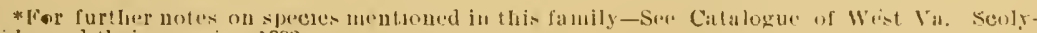
tidir and their cnemies, 1893.
} 
239. Monarthrum fasciatum, Say.

TLMBER BEETLE.

Infests Pine, White Oak, Black Oak, Basswood, Beech and Hemlock.

240. Monarthrum mali, Fitch.

TIMBER BEETLE.

Infests Pine, White Oak, Black Oak,

Red Oak. Jack Oak, Elm, Beech, Maple, Chestnut, Basswood, Honey

Locust, Yellow Poplar ('Tulip), Buckeye, Morello Cherry, Red Cedar and Hemlock.

241. Gnathotrichus retusus, Lec.

TIMBER BEEILE.

Infests White Pines; also other Pines.

242. Gnathotrichus materiarius, Fitch.

TIMBER BEETLE.

Infests Pines.

243. Pityophthorus minutissimus, Zimm.

BARK BEETLE.

Infests Black Oak, White Oak, Jack

Oak, Chestnut Oak, Dogwood.

244. Pityophthorus, sp. a.

BARK BETTLE.

Infests White Pine; also other Pine.

245. Pityophthorus pullus. Zimm.

BAKK BEETLE.

Infests Pines.

246. Pityophthorus sp. b.

BARK BEETLE.

Collected on bark of dead Maple.

247. Pityophthorus plagiatus, Lec.

Tomicus plagiatus, Lec.

BARK BE'TTLE.

Infests Pines.

248 Pityophthorus sparsus, Lec.

BARK BE'T'LE.

Infests White Pines; also other Pines. 
249. Pityophthorus cariniceps, lece.

TWIO BEETLE.

Inferts Black spruce.

250. Pityophthorus confinis, Lee.

Infests Pines.

ЉARK IEETLE.

251. Pityophthorus consimilis, Lec.

- BARK BEE'TLE.

Infests Sumach and all of the piecies of the genus Rhus.

252. Pityophthorus hirticeps, Iec.

TWIG: BEE'TLE.

Inferts Black Spruce.

253. Pityophthorus sp c.

ВАKK BEETLE.

Infests Black Spruce.

254. Pityophthorus lautus, Eich.

Inlests Pine.

BAKK BEE'TE

255. Pityophthorus puberulus, Lec.

BARK BEETLE.

Infests Pine.

256. Pityophthorus sp. d.

TWIG BEETLE.

Infests Pines.

257. Pityophthorus sp. e.

TWIG BEETLE.

Infests Pine.

258. Pityophthorus tuburculatus, Eich.

BARK BEETLF。

Infests Blick Spruce.

259. Pityophthorus sp. f.

TWIG BEETLE.

Infests Pine Twigs.

260. Pityophthorus sp. g 
261. Hypothenemus sp b.

TWIG BEETI.E.

Infests White and Black Walnut

262. Hypothenemus sp. d.

TWIG BETTLE.

Infests Pine ( $P$ inops.)

263 Hypothenemus dissimilis, Zimm.

Infests Hickory.

'TWIG BETILE.

264. Hypothenemus sp. e.

Infests Hickory.

265. Hypothenemus sp. f.

TWIG BEETLE.

Infests Oak.

266. Hypothenemus sp. g.

TWIG BEETLE.

Infests White Oak.

267. Xyloterus retusus, Lec

TIMBER BETTLLE.

Infests Large Aspen.

268. Xyloterus bivittatus, Kirby.

TIMBFR BEETLE.

Infests Black Spruce, Hemlock.

269. Xyloterus scabricollis, Lec.

Infests Pine.

TIMBER BEE'LE.

270. Xyloterus politus, Say.

Sylolerus unicolor, Eich.

TIMISER BEETLE.

Infests Beech, Black Oak, White Oak, Red Oak, Hemlock, Sugar Maple, Red Maple, Chestnut, Magnolia, Elm, Hickory, Ash, White Birch, Black Spruce.

271. Xyleborus pyri, Peck.

TIMBER BEETLE.

Infests Hemlock, Beech, Birch and Red Oak. 
2.2. Xyleborus, sp. a.

Inlestis Cedal:

THMLER HEFTLE.

273. Xyleborus obesus, 1.ee.
TIMLER BEETIE.
Infest-Black Oak, Beech and Hem- lock.

2.1. Xyleborus celsus, lijch.

Infests Hickory.

'T.MIEE ISEETLE.

27.) Xyleboruș fuscatus. Eich.

TIMER I:EETE.

Infests Black ()ak and Hichory.

가. Xyleborus sp. b.

I IIJER lBEETLE.

Infests White Oak, Chestnut ()ak, Black Oak, Jack Oak.

구. Xyleborus, sp. c.

Infests Jack Oak.

TIMJER IBETT,E.

27s. Xyleborus, sp. d.

TIMBER IBEETJ.E

Infests Black Oak. Hickory and Chestnut.

279. Xyleborus pubescens. Kimm.

TIHBEß JEE'LE.

Infest: White Oalk, Chestnut, Black Oak, Buckeye, Milgnolia, Basswood, Cultivated Cherry, Honey Locust, Jack Oak.

290. Xyleborus, sp. f.

Infests Pine.

TI.JER HEE'भ.

281 Dryocoetes autographus, Rat\%. (Drrocotes seftentrionis, Mann.)

1; $+\mathrm{RK}$ JEEETI,E

Infests Black spruce, Norway Spruce. 
282. Dryocoetes, n sp

B.IRK BEETLE.

Infests Birch (White), and Red Wild Cherry.

283. Dryocoetes affaber?, Mann.

B.LRK BEETLE.

Infests Black Spruce.

284. Dryocoetes granicollis, Lec.

BARK BEEIEE.

I 1 fests Black Spruce.

285. Tomicus calligraphus, Germ.

BARK BEETLE.

Infests all of the Pines.

286. Tomicus cacographus, Lec.

BARK BEETI,E.

Infests all of the Pines, Black Spruce, Norway Spruce.

287. Tomicus pini, Say.

BARK BEE'LE.

Infests all of the Pines, Norway Spruce.

288. Tomicus avulsus, Eich.

BAHK BEETLE.

Infests White and other Pines.

289. Tomicus cælatus, Eich .

BARK BEETLE.

Infests all of the Pines, Black Spruce Norway Spruce.

290. Scolytus quadrispinosus, Say.

HICK॥RY BARK BEETI.E.

Infests Hickory.

291. Scolytus muticus, Say.

BARK BEETLE.

Infests Hackberry.

292. Chramesus icoriæ, Lec.

BARK BEELE.

Infests Hickory.

293. Polygraphus rufipennis, Kirby.

Infests Black Spruce. 
294. Phlœotribus liminaris, Harr.

B.IRK BEETLE.

Infests Peach, cultivated Cherry, Wild Cherry.

29:.) Phlœtribus sp. b

BARK LEETIE.

Infests Red Cedar.

296. Phlœotribus frontalis, Oliv.

BARK BEETI.E.

Infests Mulberry.

297. Hylesinus aculeatus, Say.

ASH BARK BEETLE.

Infests Black Ash.

298. Hylesinus opaculus, Lec.

Infests Elm.

ELAM BARK BEETLE.

299. Phioeosinus dentatus, Say.

CEDAR BARK BEETLE.

Infests Red Cedar.

300. Dendroctonus terebrans, Oliv.

BARK BEETLE.

Infests all of the Pines.

301. Dendroctonus frontalis. Zimm.'

Infests all of the Pines, Black

Spruce, Norway Spruce.

302. Crypturgus pusillus, Gyll.

Crypturgus atomis, Lec.

BARK BEETLE.

Infests Pines, Black Spruce, Norway Spruce.

303. Hylastes sp.

BARK BEEETLE.

Infests Pines.

304. Hylurgops glabratus, Zett.

(Hylurgops pinifex, Fitch.)

BARK BEETLE.

Infests the Pines. 
Order COLEOPTERA; Family ANTHRIBID

305. Eusphyrus Walshii, Lec.

Infests dead branches on Yellow Locust.

Adults bred April 24.

Monongalia combuty.

306. Cratoparis lunatus, Fab.

Common on fungus on and under bark of dead trees.

Adults Feb. ; April 13, 17; May 9.

Wood county.

Order HYMENOPTERA; Family TENTHREDINID无.

307. Nematus robiniæ, Forbes

FOLIAGE FLIDEL

Feeding on leaves. Infests Yellow

Larre May 18, 21.

Lecust.

Wood and Jackson counties.

308. Nematus sp. a.

FOLIAGE FEETER.

Feeding on leaves. Infests Yellow

Larve May 30. I ocust.

Monongalia county.

309. Nematus sp. b.

FULIAGE FEEHER.

Feeding on leaves common. Intests

Larvie July 12.

Dogwool.

Randolph county.

310. Lophyrus pinus rigida, Nort.

FUAAE FEEUER.

Feerling on leaves. Infests frimms rigida.

Larva May 20.

Wood county. 


\section{Order HYMENOPTERA; Family UROCERIDAE.}

311. Xiphydria albicornis, IIaris.

Wris) BOKER.

Females taken ovipositing in hark

Adults June 2 ; July 7 . of deal Maple.

Monongalia comnty.

312 Urocerus abdominalis, IIarris.

WOUID BORER.

Grul, enters sap and heartwool of

Adults July 25; Ang. 30. dying trees. Infests Pine, Spruce.

Randolpll county.

313. Urocerus albicornis, Fabr.

WUOI) BORER.

Larvie enters green sap and heart wood of injured and dying trees.

Adult July 17. Infests Hemlock.

Monongalia county.

314. Urocerus cyaneus, Fabr.

WOOD BORER.

Larve bore in green and dead wood of logs and dying trees. Infests Black Spruce.

Aug. 29; Sept. 1.

Randolph county.

315. Urocerus sp. a

Adult Sept. 1.

WOOII BORER.

Flying in Spruce forest.

Randolph county.

316. Uroerus sp. b.

Adults Oct. 21.

Ovipositing in bark on living rel low Pine.

- Nononongalia county.

317. Tremex columba, Linn.

WOOI) BORER.

Larvit bores in green wood of diseased and dying trees. Hastens death of trees Infests Naple, beech.

Adults Ang. 2(); July 16; Oct. 21.

Aforongalia county. 


\section{Order HYMENOPTERA; Family CYNIPID王.}

318. Callirhytes pattoni, Bass.

GALL FLY.

Causes wooly galls on White Oak twigs.

Adults bred. Galls collected March 16 .

Preston county.

319. Neuroterus noxiosus, Bass.

GALL FLY.

Causes galls on Oak twigs.

Adults April 1. Larva Feb. 20.

Wood county.

320. Neuroterus, sp.

GAI.L FLY.

Causes galls on Oak twigs.

Adults February 24. Larva. December 24.

Wood county.

321. Ceroptres, sp.

Adults February 20.

GALL, FLY.

Causes galls on Oak twigs.

Wood county.

322. Synergus lignicola, O. S.

GALL FLY.

Causes large woody galls on Scarlet Oak twigs and branches.

Adults. Larvæ April 12

Upshur county.

Order HYMENOPTERA; Family EVANIID丑

323. Aulacus abdominalis, Cress.

BARK BORER.

Bred from Hemlock bark. Infested by Melancpliila fulvaguttata.

Adults July 24. Bred Adults April 13; May 15; .Tune 15. Grant and Monongalia counties.

Order HYMENOPTERA; Family ICHNEUMONID Æ.

324. Adelognathus briviceps, Ashm. n. sp PRIMARY PARASTTE.

Bred from Saw Fly larvae. Nematus

Adults May 30.

st. $a$. on Yellow Locust leaf.

Monongalia county. 
325. Rhyssa albomaculata, Cress.

PRIMAKY PARASITE.

Adults July 9 ; Sept. 1.

limerging from dead spruce tree infested by Unocerus cyaneus.

Randolph and Wood counties.

326. Thalessa atrata, Fabr.

PRIMA RY PARASITE.

Females taken with ovipositors inserted in wood infested by Tremex columba in dying Beech.

Adults June 20; July 14, 29. Pupæ Feb. 15. Larvie Oct. 21. Webster, Tucker and Wood counties.

327. Thalessa lunator, Fabr.

PRIMARY PARASIIE.

Females taken with ovipositor inserted in wood infested by Tremex columba larva.

Adults March 1; July 29. Pupæ Feb. 15. Larvæ Oct. 21.

Wood county.

328. Thalessa nortoni, Cress.

Female taken with ovipositor inserted in Spruce log. No trace of larvæ of any kind could be found in

Adult July 7. the wood of the log.

Grant county.

329. Ephialtes gigas, Walsh.

Collected on bark of dead Hickory and Chestnut infested by Longicorn and Buprestid larræ.

330. Pimpla conquisitor, Say.

PRIMARY PARASIIE.

Attacks Lepidopterous larvie on

Adults bred June 15 . Buckeye leaves.

Monongalia county.

331. Pimpla inquisitor, Say.

PRIMARY PARASITE.

Attacks Orgyia leucostigma on Maple. Adults bred Jan. 1; Feb. 24; Oct. 2; Nov 13.

Monongalia county. 
332. Glypta leucozonata, Ashm.

PRIMARY PARASITE.

Attacks Lepidopterous larvæ on Su-

Adults bred Oct. 24. mach leaves.

Monongalia county.

333. Euxorides americanus, Cress.

Collected on bark on dying Pine tree infested by Scolytid aud Cerambycid

Adults June 23. larvie.

Pendleton county.

334 Xylonomus albopictus, Cress.

PRIMARY PARASITE.

Attacks Buprestid larvæ and Agrilus

Adults bred July 7, 27. larve in Beech bark.

Tucker and Wood counties.

335. Xylonomus insularis, Cress.

Collected on Hemlock bark infested

Adults July 12.

by Melanophila fulvoguttata.

Grant county.

Order HYIMENOPTERA; Family BRACONID \#.

336. Bracon aguili, Ashm.

PRIMIARY PARASITE.

Attacks Neoclytus erythrocephalus larvae in Hickory and Cerambycid larvae in Apple and Sassafras.

Adults May 8, 18; July 20; Sept. 14. Bred Pupae and cocoons A pril 15, 25. Adults emerged May \&, 18.

Monongalia and Wood counties.

33i. Bracon eurygaster, Brulli.

PRIMARY PARASITE.

Attacks Cerambycid larvæ in Elm.

A ugust 4 .

Kanawha county.

338. Bracon mavoritus, Cress.

PRIMARY PARASITE.

Attacks Tetropium cinnamopterum in Spruce wood.

Adults bred Feb. 24; A pril 20; July 8. Larvie Dec. 24. Monongalia county. 
339. Bracon pectinator, Siy.

PRIMARY P'ARASITE.

A ttacks Melanophila fulioguttuta larvil in Hemlock, ('hrysobothris femorata in Oak and Cerambycid larva in Black Spruce and Elm.

Adults bred March 30 ; April 20; July 12 ; Aug. 6. Larve March :30, 31 Cocoons April 9; July 24.

(irant, Wood and Tucker counties.

340. Bracon pirsodis, Ashm.

PRIMARY PARASITE.

Attacks Dendroctonus frontalis in Pine Bark. No. 82 Catalogue $\mathrm{W}$. Va. Scolytidae, 1893.

341. Bracon simplex, Cress.

PRIMARY PARASITE.

Attacks Buprestid and Cerambycid Adults Aug. 29 to Sept. 1; July 7.

larvae in Beech and Spruce bark.

Randolph and Grant counties.

342. Bracon sp. a. Larva.

PRIMARY PARASITE.

Attacks Asemum moestum larva in

Larvae July 7. Black Spruce bark.

Grant county.

343. Bracon sp. b. Larva.

PRIIIARY PARASITE.

Attacks Melanophila fulvoguttata in

Larva March 29.

Hemlock bark.

Tucker county.

344. Phanomeris sp.

PRIMARY PARASITE.

Attacks Leyidopterous leaf roller Adult bred from coconn Aug. 8 larva on locust leaves.

Monongalia county.

345. Rhysipolis biformis Ashm.

PRIMARY PARASIIE.

Attacks Cerambycid and Buprestid larve in bark of Spruce.

Adults March 31. Bred cocoons Sept. 1. Adults Sept. 14. Monongalia and Grant counties. 
346. Rhyssalus pityophthori, Ashm.

With Pityophthorus sp d. in Pine bark. No. 83 Catalogue W. Va. Scolytidæ 1893.

347. Spathius brachyrus, Ashm.

PRIMARY PARASITE.

Attacks Pissodes strobi in Pine and Dryocoetes autographus in Spruce.

Bred Adults Sept. 28; Nov. 10. Cocoons March 15. Adults emerged April 6.

Wood and Monongalia counties.

348. Spathius brunneus, Ashm.

PRIMARY PARASITE.

Attacks Scolytus muticus in Hackberry bark. No 91, Catalogue W. Va. Scolytidæ 1893.

349. Spathius claripennis, Ashm.

PRIMARY PARASITE.

Attacks Polygraphus rufipennis in Spruce bark. No 84, Cat. W. Va. Scolytidæ 1893.

350. Spathius canadensis, Ashm.

PRIMARY PARASITE.
With Phloeosinus dentatus, in Red
Cedar; Tomicus cacographus in White
Pine and Agrilus otiosus in Uickory
bark.

Adults March 31; April 14, 18, 23, 29; May 10.

Wood county.

351. Spathius pollidus, Ashm.

\section{PRIMARY PARASITE}

Attacks 7 omicus cacographus in Pine bark. No. 89 in Catalogue West Virginia Scolytidæ 1893

352. Spathius simillimus, Ashm.

PRIMARY PARA SITE.

Attacks Buprestid larvae and Chrysobothris pusilla in Spruce. Cryptorhynchus ferratus in Sassafras, Agrilus bilineatus in Oak.

Cocoons March 26, 29, 31; May 2, 4. Adults May 1, 18, 25 ; April 25.

Grant, Tucker and Wood counties. 
353. Spathius tomici, Ashm.

$$
\text { PRIMARY PARASIIE. }
$$

Attacks Dryococtes autographus in Spruce No. 90 in Catalogue West Va. Scolytidae 1893.

354. Spathius unifasciatus. Ashm.

PRIMARY PARASI'JE.

Attacks Scolytus quadrisfinosus in Hicknry bark. No. 85 Catalngue W. Va. Scolytidae

355 Spathius, sp.

PRIMARY PARASITE.

Attacks Bupresticl larvae in Elm bark.

Bred cocoons April 13. Adult April 14

Wood county.

356. Lysitermus scolyticida, Ashm.

Attacks Scolytus 4-spinosus in Hickory bark. No. 92 Catalngue West Va Scrlytidae, 1893.

357. Lysitermus (?) sp.

Attacks Tomicus Plagiatus in Pine bark. No. 93 Catalogue West Va. Scolytidae, 1893

358 Cænophanes anthaxia, Ashm.

PRIMARY PARASITE.

Attacks Anthaxia virdicornis in Willow bark; Agrilus larvæ in Dogwood and Copturus binotatus in Sumach.

Bred cocoons April 14; May 4. Adults emerged May 24, 26. Cocons collected June 15. Adults bred Nov. 8.

Wood and Marion counties.

359. Cænophanes pityophthori, Ashm.

PRIMIARY PARASITE.

Attacks Pityophthorus sp.c. and Poly: graphus rufipennis in Spruce bark. No. 94. Catalogue West Va, Scolytidæe 1893. 
360. Cænophanes hylotrupidis, Ashm.*

PRIMAKY PARASITE.

Attacks Cerambycid larva in Cedar bark.

Bred adult April 4.

Wood county.

361. Cænophanes sp.

PRIMARY PARASITE.

Attacks Agrilus egcuus in Yrellow Locust bark.

Adults April 25. Cocoons March 16. Monongalia county.

362. Pambolus bifasciatus, Asm. (n. sp.) $\dagger$ PHINARY PARASITE.

Attacks Anthavia virdicornis in Wil-

Adults May 4; June 24. low bark.

Wood county.

363. Doryctes erythromelas, Brulle.

P'RIMARY PARASITE.

Attacks Wood-boring larvae. Kind

Bred Adult April 3 of wood not noted.

Wood county.

364. Rhogas intermedius, Cress.

PRINARY PARASITE.

Attacks Acronjeta americana on Maple.

Bred Adults Oct. 5. Collected Sept.

Monongalia county.

\subsection{Apanteles sp.}

Adults bred in October.

PRINARY PARASITE.

Monongalia county.

Attacks Orgyia leucostigma:

366. Microdus laticinctus, Cress.

PARASITE.

Collected on Locust leaves infested

Adults May 20; July 16.

by Lepidopterous and other larvae.

Wood county. 
367. Perilitus gastrophysae, Ashm.

$$
\text { J.IRAMITr. }
$$

collected on locust leares infesterl with lepidoptroms, Coleopterous and other larvate.

Arlult June 16.

Epshur county.

365. Blacus longicandus, Prov.

PRIMARY PARASITE.

Attacks lictus striatus in dry Yw.

Adults June 23.

low Locust wond.

Pocahontas county.

369). Helcon legator, Say.

$$
\text { PRHMAKY PAKASITE. }
$$

W ith Scolvetus Muticus and Agrilus

Concoms May 2. Arlults bred.

lare in Hacklerry bark.

Wood county.

:i0. Helcon occidentalis, Ashm (n. sp)*

$$
\text { PUIMAKY IRRASIIR. }
$$

Attacks Tierofumm cinnomopterem in Black sprues woml.

Bred Cocoon July S. Adult emerged July 14.

(rriant county.

371. Promachus rubiceps, Ashm.

PHIMARY PARASITE.

With Veclitus erpthrocephalus and

Adults April 24.

Agralus egenus in Locust twigs.

Monongalia county.

372. Diaspasta (Alysia) nibicunda, Say.

P.ARASITE.

Collected on Locust leaves infested Adults September 14; June 27.

by Lepidopterous and other insects.

Monongalia county.

373. Cosmophorus Hopkinsii sp. n., Ashm. MS.

Attacks Polygraphus rufipennis in Spruce bark. No 96 Catalógue W.

Va. Scolytida, 1893.

*Described by $\mathrm{W}$ m. H. Ashmead Canadian Entomologist, Vol. AXV., p. is. 


\section{Order HYMENOPTERA; Family CHALCIDI $Æ$}

374. Simcra canadensis, Cress.

PRIMARY PARASITE.

Attacks Magdalis olyra in Hickory bark.

Bred-Larvae April 23. Adults emerged May 5.

Wood county.

375. Perilampus hyalinus Say.

PRIMARY PARASITE.

Attacks Lyctus Striatus in dry Yel-

Adults June 23. low Locust wood.

Pocahontas county.

376. Eurytoma auxiceps, Walsh.

Adults March 16.

PRIMARY PARASITE.

Attacks gall insect on oak.

Marion county.

377. Eurytoma sp. d

PRINARY PARABITE.

Attacks Tomicus plagiatus in Pine bark. No. 101 Catalogue West Va. Scolytidæ 1893.

378 Eurytoma sp. e.

PRIMARY PARASITE.

Attacks Phloesinus dentatus in Red Cedar bark. No 102 Catalogue W est Va. Scolytidae 1893

379. Eurytoma sp. f.

PRIMARY PARASITE.

Attacks Polygraphus rufpennis in Black Spruce bark. No. 103 Catalogue W. Va. Scolytidae 1893.

380. Eurytoma sp. g.

Bred adult April 29.

With Agrilus otiosus in Hickory bark.

Wood county.

381. Eurytoma sp. h.

Adults May.

PRINIARY PARASITE.

Bred from galls on White Oak twigs.

Wood county. 
382. ?Diomorus Zabriskii, Cr.

In Nines of Hymenopterous insect in outer Pine bark.

Adults May 3.

Hardy county.

38: Torymus sp. a.

Adults June 23.

Bred from galls on Willow and from Spruce bark infested with Scol tirs.

Wood county.

384. Lochites sp. a.

$$
\text { PRIMARY PARASI'IE. }
$$

Attacks Poly'yaphus rufipennis in Spruce bark. No. 104 Catalogue West Va Scolytidie 1893.

38.5. Lochites sp. b.

With Scolytids in Pine bark. No. 105 Catalogue West Va. Scolytidæ 1893.

396. Lochites sp. c.

Adult May 30 .

Monongalia county.

387. Lochites sp d.

Collected on Locust leaves with leaf gall and other insects

PRIMARY PARASITE.

Attacks Dendroctomus frontalis in Pine bark. No. 106 Catalogue West Va. Scolytidæ 1893.

388. Lochites sp. e.

\section{PRIMARY PARASITE}

Attacks Tomicus calatus in Norway Spruce bark. No. 107 Catalogue W. Va. Scolytidæ, 1893.

389. Eupelmus, sp. a.

Adults April 1.

Bred from Hemlock bark infested with Melunophila fulroguttata and Aulacus abdominalis.

Monongalia county. 
390. Pteromalus sp a.

PRIMARY PARASIL'E,

Attack: Cecidomyia rubinse in Yellow Adults May 21 ; September 18.

Lncust leaf galls.

Monongalia and Jackson county.

391. Pteromalus(?) sp. b.

PRIMARY PARASITE.

Attilckis Phloesinus dentatus in Red Cedar bark. No. 109 Catalogue W. Va Scolytirlat, 1893.

392. Meraporus sp.

SECONDARY PARASIIE.

With Pimpla inquisitor pupe in coconns of Orgyza leuiostigma on Maple.

Adults February 14; November 6.

Monongalia county.

393. Gitognathus, sp. a.

Common on Yellow Lncust leaves infe-ted with leaf galls Cecidom,ia robinice and leaf miner Odontota

Adults May 20, 21, 25, 30. dorsalis.

Wood county.

394. Gitognathus (?) sp. b.

Adults July 14

Monongalia county.

395. Heydenia unica, Cook.

Attacks Dendroctonus jrontalis in Pine bark. No. 111 Catalngue West Va Scolytidae 1893.

396. Metastenus acanthocini, Ashm.

Adults March 29.

PRIMARY PARASITE.

Attacks Odnntota dorsalis larvae in mines in Yellow Locust leaf.

Wood county. 
397. Hippocephalus nulbilineatus, $1 \mathrm{shm}$.

$$
\text { P'RIMARI I'ARAAITH. }
$$

Bred from I'ine leaves infested with leaf miner Gilcrha pinifoliclla.

Adults bred July 13; May' 20.

Wood county.

39.. Spintherus sp.

Attacks L'olyoraphus rufifennis in spruce bark No. 112 Catalogue IV. Va Scolytide 18.93

399. Trigonoderus sp.

Atlateks Polygraphus rufipennis in - mruce bark. No. 113 Catalogue $1 V^{*}$ est Ta. Scolptida 1893.

400. Omphale bicinchis. Ashm.

On Locust leaves infested by Odontota dorsalis.

Adults May 18. . June 26.

IVood county.

401. Tetrastichus sp. a.

SECONDARY PARISI'E.

Attacks Thanasimus larve.

402 Tetrastichus sp. c.

Adults Jan. 28.

Monongalia county.

403. Tetrastichus sp. d.

SECONIDARY PARASITE.

Bred from Apantcles sp., cocoon on Apatcla Americana (?) larva

Adults bred Jan $10 . \quad$ Larva Nov. 13.

SECUNDARY PAKASLTE.

Attacks Pimpla mquisitor in chrysa-

lis of Orgyia leucostigma.

Marion county.

404. Tetrastichus racemariæ, Ashm.

Bred from Oak galls infester with

Adult Feb. 20 .

Veuroterus noxiosus.

Wood county. 
405. Trisholcas euschiste, Ashm.

$$
\text { EgG PARASITE. }
$$

Bred from Hemiptercus egg in

Adults June 22, 27. locust leaf.

Wood county.

406. Bruchophagus sp

Adults May 30.

Collected on Yellow Locust leaves infested with Odor tota dorsalis.

407. Decatoma sp

Collected on Spruce logs infested with Polygraphus rufipennis. No. 115 Catalogue W. Va. Scolytidae, 1893.

408. Lathromeris scutellaris, Ashm. MS.

EGG PARASITE.

Adults bred September 27.

Attacks Lepidopterous eggs on Wild Cherry twigs.

Monongalia county.

409. Chalcid, (Genus Nov. ?)

FRIMARY PARASITE.

Attacks adult Pityophthorus minutis simus in Oak bark. No. 114 Catalogue West Va Scolytidæ, 1893.

Order HYMENOPTERA; Family PROCTOTRUPID丑.

410. Aphalonomia hyolinipennis, Ashm.

With Hypothenemus sp. $b$. in White Walnut. No. 116 Catalogue West Va. Scolytidæ, 1893.

411. Leptacis sp. a.

Collected on Yellow Locust leaves.

Adults May 18

Wood county.

412. Leptacis sp. b

Adults May 21.

Collected on Locust leaves

Wood county. 
Order HEMIPTERA; Sub-order HOMOPTERA, Family COCCID正.

(Scale Insects.)

413. Undetermined species.

On Pine twigs.

413a. Pseudococcus aceris (?) Geof.

On Soft Maple and Yellow Locust.

$413 \mathrm{~b}$ Pulvinaria innumerabilis.

On Soft Maple and Yellow Locust.

414. Undetermined species.

On Yellow Locust.

Family APHIDID无.

(Plant Lice.)

415. Undetermined sp. a.

On Hawthorn twigs

416 Undetermined sp. b.

On Box Elder twigs and leaves.

417. Undetermined sp. c.

On Yellow Locust twigs

418 Undetermined sp. d.

On Yellow Locust twigs.

419. Pemphigus aceris, Morell.

On Maple leaves.

420 Pemphigus acerifolii, Riley.

On Maple leaves.

421. Undetermined sp. e.

On Pine twigs.

422 Undetermined sp. f.

On White Pine leaves.

423. Undetermined, sp. $\mathrm{g}$.

On Pine bark.

424. Undetermined, $\mathrm{sp} \mathrm{h}$.

On Balm of Gilead. 


\section{Family IMEMBRACID \&.}

(Tree Hoppers.)

425. Thelia, sp. a.

On Yellow Locust twigs feeding on sap.

Adults April 27; Aug. 8; Oct. 3.

Monongalia county.

426. Thelia, sp. b.

Adults and nymphs feeding on sap of Yellow Locust twigs.

Adults June 23, 27; July 16 . Nymphs June 27.

Wood and Hancock counties.

427. Tetamona (?) sp.. a .

Adults June 27.

Collected on Yellow Locust leaves.

Wood county.

428 Ceresa bubulus

Adults May 18; June 26, 27.

Frequent on Yellow Locust lèaves and twigs.

'Wood county.

429. Amphiscepa bivittata, Say.

Collected on Locust leaves and tender branches

Adults Ang. 8.

Monongalia county.

430. Amphiscepa sp. a.

Adults August 16; September 14 .

Collected on Yellow Locust leaves. Monongalia county.

431. Amphiscepa sp. b.

Adults September 14

Collected on Y'ellow Locust leaves.

Monongalia county.

432. Enchenopa binotata.

Common feeding on sap of tender twigs on Yellow Locust.

Adults June 24, 25, 26, 27; July 16; August 8 Nymphs June 26 


\section{3:i. Ophiderma salamandra, Fairn}

Conmon on tender twigs on Yellow

locust, feeding on saf and attended loy ants.

Adult: June 13, 27; Augrust 19. Nymplis August S, 19.

Monongalia county.

434. Smilia sp.

Arlults June 27; October 4.

Collected on Yellow Locust leaves.

Monongalia county.

\section{Family CERCOPID无.}

435. Aphrophora quadrangularis.

Collected on Yellow locust leaves

Adults October 4. and tender twigs.

Monongalia county.

436 Undetermined sp. a.

SPITTLE INSECT

Common on Pine, Spruce, Hemlock

June and July '93. and Balsain Fir.

Pocahontas, Randolph and Greenbrier counties.

437. Undetermined sp. b.

Nymphs July 11.

SPITTLE INSECT.

Common on Black Spruce twigs.

Tucker county.

435. Undetermined sp. c.

Nymphs May 20.

Common on Yellow and Scrub Pine twigs.

Wood county.

\section{Family JASSID Æ.}

439. Undetermined sp a.

Adults June 13; Oct 4.

On Yellow Locust leaves.

Nonongalia county.

440. Undetermined sp. b.

Adults June 12.

Collected on Yellow Locust leaves.

Monongalia county. 
441. Undetermined sp. c.

Adults June 24.

Collected on Yellow Locust leaves.

Wood county.

Order HEMIPTERA; Sub-order HETEROPTERA.

Family REDUVIID正.

(Bugs.)

442. Sinea diadema, Fab.

Adults May 18, 24.

Collected on Locust leaves

Wood county.

Family PHYIMATID无.

443. Phymata Wolffii.

PREDACEOUS.

Common on Yellow Locust and other

Adults Aug. 9; Oct. 8. trees, feeding on insects.

Monongalia county.

444. Phymata sp. a.

Adults Aug. 8.

PHEDACEOUS.

Collected on Locust leaves.

Monongalia county.

Family TINGITID A.

445. Corythuca arcuata.

Common on under side of Sycamore leaves, feeding on juices of leaf.

All stages June 10, 13.

Causes leaves to turn yellow.

Monongalia aıd Harrison counties.

\section{Family CAPSIDA.}

446. Calocoris sp. a.

Adults June 10.

Collected on Yellow Locust leaves.

Monongalia county.

447. Calocoris rapidus, Say.

Adults June 10; Aug. 8.

Collected on Yellow Locust leaves.

Monongalia and Harrison counties. 
448. Calocoris, sp. b.

All stages very common on Yellow Locust leaves and tender twigs Probably feeding on their juices.

Adults June 1, 10, 24, 27; July 16. Nymphs June and July Wood, Hancock and Monongalia counties.

449. Calocoris, sp. c.

Aitult June 15.

Collected on Yellow iocust leaves. Harrison county.

\section{Family LYG开ID无.}

4:0. Undetermined species a.

Adults Aug. \&.

Collected on Yellow Locust leaves.

Monongalia county.

\section{Family COREID丑.}

451. Acanthocephalina, sp. a.

Adults Oct. 3

Collected on Yellow Locust leaves.

Mono:igalia county.

\section{Family PENTATOMIDAE.}

452. Acanthosoma nebulosa, Kirby.

Adult .June 10.

Collected on Yellow Locust leaves.

Monongalia county.

453. Nizara hirlaris, Say

Adults August 6.

Monongalia county.

454. Podisus sp. a.

Collected on Yellow Locust leaves.

Monongalia county.

455. Podisus? sp. b.

PREDACEUUS.

Feelling on leaf roller larva on Yellow Locust ltaf.

June 13, feeding on larrie.

Nymplss Os:tober 4

PREDACEUUS.

Nymphs feeding on Aphids on Yellow locust twigs.

IIonongalia county. 
4iti. Podisus? sp e.

PREDACEOUS.

Frerling on Geometrid larvat and Yellow Locust leaves.

Arlult: Augrust 7. Eggs May 22.

Monongalia county.

4.7. Podisus spinosus, Dallas.

PREIUACEUUS.

Feerling on Orgyia leucostgma larvæ

Adults Sipteniber 18 on Locust laves

Monongalia county.

\section{Order LEPIDOPTERA.}

Family HESPERID王.

458. Eudamus tityrus, Fabr.

Larvæ common feeding on Yellow Locust leaves.

Adults May 4; June; Larvæ August; October 4; Eggs June, A ugust.

Wood and Monongalia counties.

\section{Family LIPARID正}

459. Orgyia leucostigma, S \& A

Larve common feeding on Maple and frequent on Yellow Locust.

Pupk June 28; Larve June 26; September 22.

Monougalia and Wood counties.

\section{Family LIMACODID无.}

460. Empretia stimulea, Clem.

Larre feeding on Yellow Locust leaves, also Blackberry leaves.

Larvie August 8.

Monongalia county.

461. Limacodes sp.

Larve A uerust 15.

Larra feeding on Yellow Locust leaves.

Nonongalia county. 


\section{Family PSYCHID 现}

46.. Thyridopteryx ephemeræformis, Steph

Observed Cocoons on Red Cedar.

\section{Family SATURNIIDAE.}

46:i. Attacus promethea, Dru

Larvir eommon feetling on "Vellow Poplar" (Tiulip Tree) Sassafras, Wild Cherry, and P'ersimmon leares. Adults June; Larva .June: August 15.

Wool and Monongalia counties.

164. Attacus angulifera, Walk.

Larve olsserved feeding on leaves of Tulip tree.

Adults bred June 16 (1S-4.) Jackson eounty.

465. Attacus cecropia, Linn

Larve frequent feelling on leaves of a variety of a forest shade and fruit trees. Observed on Maple, Persimon, Hickory, ete.

$465 a$ Actias Iuna, Linn.

Larva common on Hickory, Walnut and Sweet Gum.

466. Telea polyphemus, Cram,

Larve common feeding on Oak leaves, Taken also on Elm and

Adults A ugust 2. Maple.

Firyette county.

467. Hyperchiria io, Fab.

Arlults June 13.

Larva observel on Hickory and Maple.

W nod county.

\section{Family CERATOCAMPID无。}

46s. Eacles imperialis, Dru.

Larve common on Maple and Syeamore.

Adults July 31.

Monongalia and Kanawha counties. 
469 Citheronia regalis, Fit).

Ailults June.

Nonongalia county.

470 Anisota senatoria, S. \& 4

Larva common on Black and Scarlet Oaks, defoliating trees.

Aclults July 12. Larvæ Sept. 10.

Larva frequent feeding on leaves of Walnut, Hickory and Persimmon. Wood county.

471. Dryocampa rubicunda, Fab.

Larva common feeding on Solt Maple leaves.

\section{Family BOMBYCID正.}

472. Hemileuca maia, Dru.

Larvæ frequent feeding on White Oak leaves.

Arlulta October. Larvæ May 20.

Wood and Jackson counties.

\section{Family COSSID无.}

473. Prionoxystus robiniæ? Peck.

WOOD BORER.

Larva common in wood of living Yellow Locust trees, alsu Oaks. Very injurious to wood.

Alults emerging June 13. Larvie collected June 27. IVood and Monongalia counties

474. Cossus sp.

Larvæ common in wood of living Oak trees. Very injurious to wood. Causes large black holes one-half to one inch in diameter.

\section{Family NOCTUIDÆ.}

475. Hydrocia nitula, Gn.

Larvie boring in tender sprouts of Yellow Locust and Maple. 
Family GEOMETRID王.

476. Ennomos magnarius, Gn.

Adults bred Octoler 3 .

Larva and Pupa on Maple.

Monongalia county.

\section{Family TORTRICIDÆ.}

477. Lophoderus politana, How.

PINE TUBE BULLER.

Larve feeds on Pinc leaves. Forms tubes of the stumps of green leaves.

\section{Family GRAPHOLITHID王.}

478. Retinia Comstockiana, Fern.

Larve bore in twigs and small branches on living Pitch Pine trees. Causes balls of turpentine to form

Larvie June 27. where they enter the bark.

Wood county.

479. Ecdytolopha insiticiana, Zell.

LUCUST TWIG BORER.

Larva bore into the tender sprouts and twigs of Yellow Locust. Very injurious to young trees. Common.

Larve June 13; August 10; October 3.

Monongalia county.

\section{Family GELECHIID丑.}

480. Depressaria robiniella (?), Pack.

Larve feeding on Yellow Locust

Larvie July 30. leaves.

Wayne county.

481. Gelechia pinifoliella, Cham.

P1NE LEAF MINER.

Common in Yellow and Pitch Pine leaves. Causes tips of leaves to turn brown.

Pupre May 20. Bred adults July 13.

Hampshire, Hardy, Grant and Wood counties. 


\section{Family LITHOCOLLETID E}

482. Lithocolletes robiniella, Clem.

LUCUS'T LEAH MINER.

Larva form blisters on under side of Yellow Locust leaves. Common.

Larvæ August 8.

Monongalia county.

\section{Order DIPTERA; Family CECIDOIMYID AE}

483. Diplosia resinicola, O. S.

Observed.

484. Diplosia pini-rigidæ, Pack.

485. Cecidomyia robinæ, Hald.

Common and destructive Infests tender leaves causing galli on their Aclults bred. Larvæ June, July.

Wood, Jackion, Hancock and Monongalia counties.

\section{Family SYNPHID EE.}

4S6. Undetermined sp. a.

Larva feeding on aphids on Yellow Locust.

Larvæ August 8.

Monongalia county.

487. Undetermined sp. b.

Larva October 4.

Monongalia county.

Larve feeding on aphids on Yellow Locust.

\section{Family TACHINIDE.}

488. Tachina orgyia, Twns.

Attacks larve and pupe of Orgyia leugcostigma on Maple, Yellow Locust, etc. Common.

Aclults bred October 2. Noveinber 5 . 
4५\%. Undetermined sp. a

Chrysalids frequent in mines of .Monohanus scutellatus in White Pine.

Chrysalids collected May 5.

Pendleton county.

490. Undetermined sp. b.

Attacks Thanasimus dubius adults on Pine.

Bred larvar August 12. Pupar August 1\%. Adult emerged A ugust 28.

Monongalia county.

\section{Family Anthomyiidæ.}

491. Anthomyia sp.

Adults Oetoler 3 .

Alult feeding on aplicds on Yellow Locust leaves.

Monongalia county.

\section{UNDETERIINED.}

492. Dipterous sp. a.

Bred from larva taken in mines of Polygraphus rufipennis larve under Silutuce bark.

Adult bred. Pupe May S. Larva March, Augu-t and Septemiber.

Randolph, Grant and Tucker counties.

493. Dipterous sp. b.

Larvac and cocoons common in mines of Polygraphus rufipennis larvae in Spruce bark. Common. Adults bred April. Larvae and cocoons March, May, Iune, August, September.

Grant, Tucker and Randolph counties.

494. Dipterous larvæ sp. c.

Larvae March 29.

In mines of Polygraphus rufipennis in Spruce bark.

Tucker county. 


\section{LIST OF FA M I IES.}

\section{COLEOPTERA.}

Carabidae 1-S,

Staphylinidae 9-14,

Phalacridae 15,

Cory ophidae 16,

Coccinellidae 17-21,

Endom ychidae 22,

Colycliidae 23-28,

Cucujidae 2933 ,

Mycetophagidae 34-35,

Histeridae 36-42.

Nitidulidae 43-52,

Lathridiidae 53-56,

Trogositidae 57-61,

Elateridae 62-64,

Buprestidae 65-88,

Lampyridae 89-91
Malachidae 92,

Cleridae 93-111,

Ptinidae 112-116,

Lymexylidae 117-121,

Scarabaeidae 122-127,

Spondylidae 128 , Cerambycidae 129-175,

Chrysomelidae 176 202,

Tenebrionidae 203-209,

Melandryidae 210-211,

Pythidae 212-214, Attleabidae 215,

Curculionidae 217-231, Brenthidae 232-235, Scolytidae 236-304, Anthribidae 305-306.

\section{HYMENOPTERA.}

Tenthredinidae 307-310,

Uroceridae 311-317,

Cynipidae 318-322,

Evaniidae 328 ,
Ichneumonidae 324-335, Braconidae 336-373, Chalcididae 374-409, Proctotrupidae 410-412.

\section{Order HEMIPTERA Sub-order Homoptera.}

Coccidae $413 \cdot 414$ A phididae 415-424,

Membracidae 425-434,
Cercopidae 435-438,

Jassidae 439-441.

Order HEMIPTERA Sub-order HETEROPTERA.

Reduviidae 442,

Phymatidae 443-444,

Tingitidae 445 ,

Capsidae 446-449.
Lygaeidae 450,

Coreidae 451,

Pentatomidae $452-457$. 


\section{LEPIDOPTERA.}

Hesperidae 458 ,

Liparidae 459,

Limacodidae 460-461,

Psychidae 462,

Saturniidae 463-467,

Ceratocampidae 468-471, Bombycidae 472,

Cecirlom yidae 4\$3-485, Symphidae 486-487,
Cossidae 473-474,

Noctuidae 475 ,

Cieometridie 476, Tortricidae 477,

Grapholithidac 478.479,

Gelechiidae 480-481,

Iithocolletidae 482.

\section{DIPTERA.}

Tachinidae 488-490, Anthomyiiclae 491. 


\section{INDEX TO INSECTS}

Acanthocephalinasp. a. 451 $\lambda$ can thooman ebulosa 452 Acmaesdera ornata 79 Acmaeodera culta 80 Acronycta americana 364 Actias lima $465 a$ Adalia bipunetata 20 Adelognathus briviceps 324 Arlimonia cavicollis 195 Adynerus sp. \%) Agrilus bilineatus 83, 352. egenus 86,361. 371 fallax 84 otiosus 82, 93, 350, 380 politus 85 sp. b. 87 sp c 88 larvae $334,358,369$

A laus nculatus 63

Alonium paralielopipedum 24 Amphiscepa bivittata 429

$$
\begin{array}{ll}
\text { sp. a. } & 430 \\
\text { sp. b. } & 431
\end{array}
$$

Anatis 15-punctatus 21 A ncanthocinus obsoletus 167 Anisota senatoria 470 Anomoea laticlavia 176 Anthaxia seneogaster 73 flavimana 75 viridicornis

Anthomia 491

Anthonomus nebulosus 224 A panteles sp. 365, 402 Apatela americana larvæ 402 Apathius sp. 355

Aphalonomia hyolinipennis 410
Aphids 17, 18, 19, 20, 21, 455, $486,487,491$

Áplirophora quadrangularis 325 Apion nigrum 217 Arhopalus fulminans 145 A semumi mostum 130, 142 Attacus $\Lambda$ ngulifera 464 cecropia 465 promethea 463

Attalus scincetus 92 Attelabus nigripes 215 Aulacus parallelopipedum 24 Aulacus abdominalis 323,389 Aulonium tuberculatum 25

Bactrididium ephippigerum 61 Balaninus ca"yatrypes 231

baptolinus longiceps 12

Batyle innicollis 142

Blacus longicandus 368

Blepharida rhois 196

Bostrychus bicornis 115

bracon aguili 336 eurygaster 337 mavoritus 338 pectinator 339 pirsodis 340 simplex 341 sp. larvæe, a. 342

74, 358, Bracon larvæ sp. b. 343 362 Brontes dubius 33

Bruchophagus sp 406

Buprestis lineata 70 rufipes 69

Buprestid sp. c. 81 Buprestid larvæ 105, 329, 334, 341 $4 \quad 345,352,355$ 
Cirnophanes anthaxia 3 is hylotrupidis 360 pityoptlıori 359 sp. 361

Callidium antennatum 1:30

Callirhytes pattoni ö1s

Calocoris rapidus 147

$$
\begin{aligned}
& \text { sp. a. } 446 \\
& \text { sp. b } 44 i \\
& \text { sp. c. } 449
\end{aligned}
$$

Catngenus rufus 30

Crecidomyia robiniale 390 ,

Contronopus ralcairatus 203

('erambycid -5 ) a. 175

larva 111, $3: 39,836$. $337,393,3+1$.

Ceresil bubulu: 428 345,360

Cempteres sp. 321

Cerotomar calmineit 19):

Chalcid 409

Chalcophora campestris (i5)

Chariessa pilosa 103

Chauliognathus pennsylranicus

Chion cinctus 136

Chlaenius tricolor $S$

Chlamys plicata 177

Chramesus icoriae 93, 205, 292

Chrysomela bigsbyana 189

$$
\text { scalaris } 188
$$

suturalis 187

Chrysobothris azurea is

femorata 76,339

Citheronia regalis 469

$$
\text { pusilla } 7 \%, 352
$$

Clerid larve sp. a. 106

sp. b. 107

sp. c. 108

sp. d. 109

sp. e. 110

sp. f. 111

Clerus furmicarius 100 quadrisignatus, Say

$$
\text { var. nigripes } 96
$$

Clerus sp. a. 97

Cloeotus aphodioides 122

Cloeotus globosus 123

Clytanthus ruricola 150
Coccinella 9-notata 18 sanguinea 19

('olaspis brunnea $18: 3$ puncticollis 185 tristis 184

Colastus unicolor 4:)

Coleopterous larvid :367

('ollydium lineola 26 (i

Collidium antennatum 135

Coptocycla guttatit $20^{2}$

Copturus binotatus 230, 3i5s

39., Corthylus punctatissinus 280

485 Corticaria elongata st)

$$
\text { s) } 5.5 \text {. }
$$

Corythuca arcuata 445

('molmophorus hopkinsii 373

(C)sus s). 474

Cossonus corticola 2:35)

Cossonus plitaleal 2034

Coxelus guttulatus 23

Critoparis lunatus 306

Crepidodera helxines 195 rufipes 197

Cryptarcha ampla 47

Cryptocephalus mammifer $17 \mathrm{~s}$

Cryptorhynchus obliquus 228 ferratus 229 ,

Crypturgus pusillus 205, 302 atom us 205, 302,

Cucugus clavipes 31

Cychrus ridingsii 1

Cymatodera bicolor 94 inornata 95

Cyllene picta 64,143 robiniae 47, 4S, 49, 144.

193

Decatoma sp. 407

Dendroctonus frontalis $9.99,106$, 205,301 , 340,387 , 395

Dendroctonus terebrans 300

Dendrophagus glaber 32

Depressaria robiniella 480

Diasposta (Alysia) nibicunda 372

1)icerca divaricata 66

lurida 68

obscura 67

Diomorus zabriskii $3 S 2$ 
Diplocoelus brunneus 35

Diplosia pini-rigidae 484 resinicola 483

Dipterous sp. a. 492

sp. b. 493.

larva sp. c. 494.

Distenia undata 151

Dorcaschema nigrum 156

Doryctes erythromelas 363

Doryphora clivicollis 186

Dromius quadricollis 7

Dryocampa rubicunda 471

Dryocoetes affaber 41, 283

autographus 14,37 . 281,353

granicollis 284

septentrionis 14,37 ,

281,353

n. sp. $14,52,282$,

Eacles imperialis 468

Eburia quadrigeminata 137

Ecdytolıpha insiticiana 91,479

Elaphidion mucronatum 138

Elaphidion parallelum 139

Elasmocerus terminatus 93

Ellychnia corrusca 90

Empretia stimulea 460

Enchenopa binotata 432

Endomychus biguttatus 22

Ennomos magnarius 476

Enoplium quadripunctum 104

Ephialtes gigas 329

Epuraea avara 44

truncatella 45

Eudamus tityrus 458

Eupelmus sp. a. 389

Eupogonius vestitus 170

Eupsalis minuta 232

Eurytoma auriceps 376

sp. d. 377

sp. e. 378

sp. f. 379

sp. g. 380

sp. h. 381

Eusphyrus walshii 305

Euxorides americanus $33 ;$

Gastroidea cyanea 190

Gelechia pinifoliella 397,481

Geome trid larva 456

Gitognathus sp. a. 393

s) b 394

G'ypta leucozonatit 3.32

Glyptoselis pubsecn. 180

Griathotrichus materiarus

retusus 241

Gries oculatus 157

Graphisurus pusillus 166

Gymnetron teter 227

Heleon legator 369

occidentalis 370

Hemileuca maia 472

Hemipterous egg 405

Hemirhipus farcicularis 64

Himatium eonicum 233

Hippocephalus nulbilileatus 397

Hiplodamia convergens 17

Hister cylindricus 38 parallelus 37

Hololepta fossularis 36

Homolata sp. 9

Hoplosia nubila 168,

Hyedenia unica $3 ! 45$.

Hydroecia nitula 475,

Hylastes sp. 303

Hylecoetus larvae sp. a. 117

Hylesinus aculeatus 297

Hylobius abietes 220

opaculus 99,298

Hylur ops glabratus 304

pinifex 304

Hymenopterous insect 95, 382

Hyperchiriaio 467

Hyperplatys aspersus 163

maculatus 164

Hy pophloeus bicolor 207

cavus 204

parallelus 205

thoracicus 206

sp. a. 208

Hypothenemus dissimilis 263

sp. b. 261, 410

sp. d. 262

sp. e. 264

sp. f. 265

sp. g. 266

37,
242 
Ips fasciatus 48, 49 sanguinolentus jo

Lachnosterna hirticula 126 ; fusca 125

Latridius maculatus 53 opaculus 54

Lathromeris scutiliaris 408

Leall-roller larra 454

Lepichoptera 372

Lepturges signatus 162

Lepiclopiterous larvae 330. 332, $344,366.367,372$,

$40 \mathrm{~s}$

Leptostylus aculiferus $15 \mathrm{~S}$

Leptacis sp. a 411

sp. b. 412

Lina lapponica 191

Limacodes sp. 461

Liopus alpha 160

punctatus 161

variegatus 159

Listronotus latıusculus 2018

Lithocolletes robiniella 482

Litargus 6-punctatus 34

Lochites sp. a. 384

sp. b 385

sp. c. 386

sp. d. 387

sp. e 388

Longicorn larvae 329

Lophyrus pinus-rigida 310

Lophorlerus politina 477

Lyctus striatus $116,368,375$

Lymexylon larval sp. a 119

sp. b. 120

sp. c. 121

Lymexylid larva sp. a. 118

Lysitermus scolyticida 356

Lucidata atra $8 y$ sp. 357

Macrodactylus subspino:-us 124

Magdalis inconspicua 223 olyra 222.374

Melanophila fulvoguttata 71 , $323,335,339,343$

$$
\text { sp. a. } 72
$$

Meraporus sp. 392

359

Metastenus acanthocini 396
Microdus laticinctus 366

Molorelius bimaculatus 140

Monithrum fasciatum 52, 239 mali 26,240

Nonohammus confusor 154 marmorator 15); scutellatus 153,

489

Myochrous denticollis 181

Nematus robiniae 307

sp a. 308,324

sp. b. 309

Nemosoma cylindricum 57

Neoclytus capraea 148 erythrocephalus $\mathbf{1 4 9}$,

luscus 147

336,371

Neuroterus noxiosus 319,404 sp. $32 \mathrm{C}$

Nitidulidae Jarva 49

Nizara hirlaris 453

Oberea ruficollis 174 tripunctata var, myops.

Odontota dorsalis $200,393,394$, 400,406

Odontota nervosa 201

Olibrus bicolor 15

Olophrum obtectum 13

Ourphale bicinchis 400

Oncicleres cingulata 171

Ophiderma salamandra 433

Orchestes niger 225 ephippiatus 226

Orgyia leucostigma $331,365,392$, $403,457,459,488$

Orthaltica copalina 1!99

Orthopleura damicornis 105

Otiducephalus chervolatii 221

Pachybrachys atcmarius 179

Pambolus bifasciatus 362

Paromalus difficilis 41

bistriatus 40

estriatus 39

Pavandra brunnea 1288

Paria aterroima 182

Pemphigus aceris 419 
Pemphigus acerifolii 420

Perilitus gastrophysae 367

Purilampus hyalinus 375

Phanomeris sp. 344

Philothermus glabriculus 28

Phloeotribus liminaris 108, 294 frontalis 59,296 sp. b. 295

Phloeosinus dentatus 94,96, 299, $350,378,391$

Phyllobeanus dislocatus 102

Phyllechthrus gentilus 194

Phyllodecta vulgatissima 192

Phy mata wolffii 443

Phymata sp. a. 444

Phymatodes dimidiatus 134 variabilis 133

Pimpla inquisitor 331, 392, 403 conquisitor 330

Pissodes strobi 219, 347

Pityophthorus cariniceps 249 confinis 43,250 consimilis 102,251 hirticeps $25 \%$ lautus 254 minutissimus 243

pullus $96,99,245$

plagiatus 247

puberulus 255

sparsus 248

tuburculatus

sp. a. 244

sp. b. 246

sp c. 253,35 !

sp. d. $99,256,346$

sp. e 257

sp. f. 259

sp. g. 260

Platynus angustatus 5

sinnatus 6

Platypus compositus 49, 237 quadridentatus $26,2 \overline{7}$,

l'legaderus transversus 42. 236

Podisus sp a. 454.

sp. b 455 .

sp. e. 456 .

spinosus 457.
Pogonocherus penicellatus 169 .

Polographus rufipennis, 9, 12, 40, 41, 45, 98, 99, 102, 107, $2(1) 5,206,209,293,349$, $359,373,379,384,398,399$, $407,492,493,494$.

Prionoxystus robiniae 473 .

Prionus laticollis 129 .

Promachus rubiceps 371 .

Prometopia 6-maculata 46

Pseudococcus aceris 413 a

Ptermalus sp b. 391

$$
\text { sp. a. } 390
$$

Pterostichus honestus 4 adoxus 3

Ptlinus ruficornis 113

Pulvinaria innum rabilis 4136

Pupuricenus humeralis 141

Pytho americanus 213 niger 212

Quedius peregrinus 10

Retinia comstockana 478

Rhagium lineatum 152

Rhizophagus dimidiatus 51

bipunctatus 52

Rhogas intermedius 364

Rhyssalus pityophthori 346

Rhysipolis biformis 345

Rhyssa albomaculata 325

99, Sacium biguttatum 16

258 Saperda discoides 172

Scolytus muticus 109, 291, 34\$, 369 quadrispinosus 109 , 290,354 356

rugulosus 293, 92, 102 , $108,109,385$

Scolyticls 10, 11, $22,25,26,28$, $51,333,383$

Serropalpus barbatus 211

Siagonium americanum 14

Silvanus planatus 29

Sinea diadema 442

Similia sp 484

Sinoxylon basilare 93,114

sitones flavescens 216

Smicra canadensis 374 
Smilia sp. $43 . t$

Smodicum eucujiforme $1: 2$

Spathius canadensis 350

claripemnis $: 19$

brunneus 3 is

brachyrus 347

pollidus 351

simillimus 252

tomici 353

unilasciatus 35.4

Spinth(rus sp). 39s

Sosylus costattus 27

Synergus lignicola 322

Tachys manus 2

Tachina orgyia 488

Tenebrioides castanea 60 corticalis 59

Tenebrionod larva 209

Telea poly phemus 466 ;

Tetamona sp a. 427

Tetrastichus euchiste 40:5

rucemaritu 404

sp. a. 401

sp c. 402

sp d. 403

Tetropium cinnamopterum 131 ,

Thalessa atrata 326

338,370

lunator 327

nortoni 328

Thanasimus dubius 99, 490 formicarius 100 trifusciatus 98

Thanasimus larva 401

Thancroclerus sanguineus 101

Tharops ruficornis 62

Thelia sp. a. 425

$$
\text { sp. b. } 426
$$

Thyridopteryx ephemerae formis 462

Tomaxia bidentata 214

Tomicus avulsus 106, 288

$$
\begin{aligned}
& \text { cacographus } 37,38,42 \text {, } \\
& 57,96,49, \\
& 106,205 \\
& \text { 286, } 350 \text {, } \\
& 351 \text {. }
\end{aligned}
$$

Tomicus calligraphus $34,43,56$, 99,285 caelitus :), 289, 3ss pini $9,110,205,287$ plagratus $377,377,247$

Torymus sp. a. :35:3

T'prinex columba 317, $322(;, 327$

'Trigonoderus sp. 399?

Trisholcals euschiste 40j

Trogosita viruscens 58

Urucerus abdominalis 312 albicornis 313 cyaneus $314,32 . ;$

sp. a. 315

sp. b. 316

Urograph is fasciatus 165

Valgus canaliculatus 127

Xantholinus cephalus 11

Xestobium squaliclum 112

Xiphydria albicorn is 311

Xyleborus celsus 204, 274

fuscatus 207, 275

obesus 273

pubescens 207, 279

pyri 271

sp. a. 272, 208

s]). b. 276

sp. c. 277

sp. f. 280

Nylita lacvigata 210

Xylonomus albopictus 334 insularis 335

Xyloterus bivittatus $4 \$, 49,101$. politus $26,50,270$ retusus 26 scabricollis 26 ? unicolor 26,$50 ; 270$ Xylotrechus colonus 146

\section{8}




\section{INDEX TO TREES.}

Apple,

Pyrus malus 17, 18, 19, 20, 59, 76, 89, 92, 102, 108, 109, 149, 15!4, $200,201,336$.

Ash, Mountain,

Pyrus Americana 188.

Ash, Black,

Fraximus sambucifolia 146, 147, 148, 270, 297.

Aspen, Large,

Populus grandidentata 36, 121, 267

Basswood,

Tilia Americana 49, 61, 76, 171, 237, 239.

Balm of Gilead, Beech,

Populus balsamifera var. Canadensis 20, 424.

Fagus ferruginea $16,23,34,35,47,52,53,54,60,61,62,113$, $115,128,132,137,138,162,200,232,237,239$, $240,270,271,273,317,334,3+1$.

Birch, Black,

Betula lenta 14, 52, 270, 271, 282.

Box-Elder.

Negundo aceroides 20, 21, 416 .

Buckeye,

Aesculus flava 240, 279, 330

Cedar, Red,

Juniperus Virgıniana 94, 96, 175, 218, 240, 272, 295, 299, 350, $360,378,391,462$.

Cherry, Cultivated,

Prunus oulgaris 19, 113, 240, 279, 294.

Cherry, Wild Black,

Prunus serotira 108, 113, 116, 200, 237, 294, 408, 463.

Cherry, Wild Red,

Prunus Pennsylzanica 195, 198, 282.

Chestrut,

Castanea sutiza var. Amcricana $83,118,145,146,151,165,207$, $208,231,236,237,240,270,278$. 279,329

Dogwood,

Cornus Florida 88, 140, 161, 243, 309, 358. 
Elm,

(7mus 99, 132. 137, 146. 232, 240, 164, 237, 270, 295, :337, 3:39, 355,466 .

Fir, Balsam,

Alues balsamed 436 .

Hawthorn,

Cratagus 415 .

Halkherry,

Celtis oridentalis $54,109,163,291,345,369$.

Hemlock,

Tsuga Camadensis, $71,101,119.145,203,2: 39,240,270,273,31: 3$, $32: 335,339,343,389,436$.

Hickory,

Carya, 34, 39, 68, 73, 76, 80, 81, 82. 93, 105, 109, 114, 116, 143, $146,149,171,172,204,214,222,264,271,274,275,278$, $290,292,329,336,356,3 / 4,380,465,465 a, 467,469$.

Lor'ust, Honey,

(iledip. cha triacanthos, $240,279$.

Locust, Iellow,

Kolnnia Psuedacacia, 17, 15, 19, 28, 29, 33, 36, 47, 48, 49. 55, 80, $86,91,116,124,132,149,160,164,176$, $179,181,182,183,184,185,186,187,193$ $194,19-199,200,201,202.215,216,217$, $221,227,305,307,308,824,344,361,366$, $367,368,371,372,375,386,390,393,394$, $400,405,40 \mathrm{n}, 411,412,413 \mathrm{a}, 413 \mathrm{~b}$, $414,417,418,425,426.427,428,424$, $430,431,432.433,484,435,439,440,441$, $442,443,444,446,447,448,449,450,451$, $452,453,454,455,456,457,458,459,460$, $461,473,475,479,480,482,455,486,487$, 488,491 .

Maple, sugar,

Maple,

Aier saciharimum 30, 50, $122,138,150,237,270$.

Aier. 26, 76, 113, 149, 158, 64. 240, 246 270, 317, 3:31, 364, 392, $413 \mathrm{i}, 413 \mathrm{~b}, 419,420,459,465,466,465,468,471,475$, $476,488$.

Magnolia,

Hagnolıa acuminata $120237,270,279$

Iluberry, Red

Morus, rubara 59, 64. 114, 143, 295.

Oak, White

Qu't cus alba $76,89,87,117,133,146,239,240,243,266,270$, $276,279.318,381,472$. 
Oak, Chestnut

Oak, Ried

Quercus Prinus 122, 243, 276.

Oak, sicarlet

Quercus rubra 240, 270, 271.

Quercus cociinea $322,470$.

Oak, Black

Quercus tunctora $24,26,27,39,76,83,123,136,138,139,146$, $165,207,232,236,237,239,240,243,270,273$,

Oak, Jack, $275,276,278,279,396,470$. Oak,

Quercus nigra 26, 139, 240, 243, 276, 277, 279.

Quercus 63, 67, 125, 126, 129, 141, 145, 149, 265, 319, 320, 321,

Peach, $339,352,404,409,466,474$.

Persica vulgarls 66, 127, 149, 294.

Persimmon,

Dinsprros Virginiana 126, 149, 463, 465, 469.

Pine, White

Pinus strohus 153, 212, 219, 241, 244, 218, 260, 261, 28.5, 2866.

Pine, $287,288,289,300,301.340,350,422,489$.

Pinus 9, 13, 22, 23, 25, 26, 37, 38, 42, 43, 56.57, 58, 78, 95, 96, 99, $100,106,127,129,130,135,152,155,167,180,21 \%, 220$, $235,239,240,241,242,24 t, 245,247,248,25(1,254,25.5$, $256,257,259,260,262,269,280,285,286,287,288,289$, $301,301,302,303,304,310,312,316,333,340,346,347$, $351,357,377,382,385,387,395,397,413,421,423,436$,

Plum, $438,477,478,481$.

Prunus domestica 75, 161.

Sassafras,

Sassafras officinale $229,238,336,463$.

Spruce, Norway

Picea excelsa 281, 286, 287, 289, 301, 302, 388.

Spruce, Black

Piced nigla 1, 2, 3, 4, 5, 6, 7. 8, 9, 10, 11, 12, 13, 14. 15, 28, 32, $37,40,41,44,45,48,49,51,72,77.89,90,98,99$ $102,10 \overline{7}, 112,130,131,134,154,156,166,167,209$, $210,211,212,219,235,249,252,253,258,270,281$, $283,284,286,289,293,301,302,312,314,315,325$, $326,328,338,339,341, \therefore 42,345,347,348,352,35 \%$ $359,370,373,379,383,384,398,399,407,436,437$,

Sumach, $492,493,494$.

Rhus 97, 102, 162, 169, 176, 196, 199, 215, 228, 230, 251, 332, 358. Sycamore,

Platamus occidentalis $65,177,445,468$ 
Sireet-ium.

Liquidambar Styracifna thisal.

Tulip, "Yellow Poplar"

Liviodendron 7 ulipfira $240,463,464$.

Walnut, Black

Juslans nigra 50, 82, 103, 104, 139, 140, 223, 2(it, 465a, 46! Waluut, White Juglans cunera $163,294,410,46.5 a$.

IVillow,

Trais 8.5, 149, 170, 189, 190, 191, 198, 225, 226, 358, 362, 383.

Willow, Black

Salix nigra 19:2. 
$+$ 
